\title{
K-ear: Extracting Data Access Periodic Characteristics for Energy-aware Data Clustering and Storing in Cloud Storage Systems
}

\author{
Xindong You ${ }^{1}$, Tian Sun ${ }^{1}$, Dawei Sun², Xunyun Liư ${ }^{3}$ Xueqiang Lv ${ }^{1}$, and Rajkumar Buyya ${ }^{4}$ \\ 1. Beïjing Key Laboratory of Internet Culture and Digital Dissemination Research, Beïing Information Science and Technology \\ University, Beijing, China \\ 2. School of Information Engineering, China University of Geosciences, Beïing, China
}

3. Artificial Intelligence Research Center, National Innovation Institute of Defense Technology, Beijing, China

4. Cloud Computing and Distributed Systems(CLOUDS) Lab, School of Computing and Information Systems, The University of Melbourne, Australia

* Corresponding author: Dawei Sun

\begin{abstract}
Rapid increase in energy consumption is a serious problem in cloud storage systems. Data accessed in large-scale storage systems usually exhibit temporal and spatial characteristics, which make it possible to reduce energy consumption by clustering data with similar access characteristics for storage in the same zone of cloud storage systems. Existing works usually only focus on the frequency of data access. However, widely existing phenomena show data access with seasonal and tidal characteristics in cloud storage systems. The seasonal and tidal characteristics of data access are extracted thoroughly in this paper. According to the extracted data access characteristics, energy-aware data clustering through a machine learning algorithm (K-ear) is proposed. K-ear classifies data into five seasonal categories according to their seasonal access characteristics and then classifies every seasonal category into three tidal categories according to its tidal access characteristics. The fifteen classified categories are stored in different storage zones with different energy and performance modes. Simulation experiments using CloudSimDisk with the constructed mathematic models demonstrate that the proposed K-ear algorithm is more energy-efficient than the default data clustering algorithms in Hadoop and the classical data clustering storage strategy according to the data access frequency (SEA, Striping-Based Energy-Aware Strategy)
\end{abstract}

\section{Keywords:}

Energy Consumption, Cloud Storage System, Data Clustering, Data Access Characteristics, Seasonal Characteristics, Tidal Characteristics.

\section{Introduction}

The exponential growth of the volume data is becoming one of the leading causes of high energy consumption in cloud storage systems [1][2][3]. It has been reported in the literature [4] that the energy consumed by data centers will be more than 1000 TWh during 2013-2025, which will surpass the total energy consumption of Japan and Germany. The energy consumed by data centers, including for cooling equipment, will consume $5 \%$ of the total energy consumption worldwide. An even more serious problem is that increasing energy consumption will produce high carbon and GHG (Greenhouse Gases) emissions [6][7], which will result in serious environmental pollution. Therefore, reducing energy consumption is one of the hottest issues in the cloud storage domain. Classifying data into different categories according to their access characteristics is an efficient way to enhance energy efficiency in cloud storage systems. Different data categories are stored in different storage zones, running in

This is the author manuscript accepted for publication and has undergone full peer review but has not been through the copyediting, typesetting, pagination and proofreading process, which may lead to differences between this version and the Version of Record. Please cite this article as doi: $10.1002 /$ cpe.6096 
different energy and performance modes. This kind of method will lead to less energy consumption in the storage zone, which has a low energy and performance mode while the workload is light. However, only the instant access frequency is considered in the current existing energy-aware data classification strategies. Long periodic access characteristics, such as seasonal characteristics and tidal characteristics, are not considered. Classifying data according to their instant access frequency will lead to frequent data migration, which will result in worse performance. Extracting the long periodic access characteristics is an effective solution, which could allow the low energy and performance running mode for a relatively long time. On the other hand, seasonal and tidal access characteristics obviously appear in cloud storage systems. As shown in Fig.1, the access frequencies (search index) of spring clothing, summer clothing, autumn clothing and winter clothing exhibit obvious seasonal period characteristics. Other words with seasonal characteristics also have seasonal periodic access frequency.

As Fig 1 is the screenshot from the Baidu website, there are Chinese words in the figure. We list the Chinese words in the Fig.1 from left to right, and top to down. where “春装”means “spring clothings”, “夏装”-“summer clothings”, “秋装”-“autumn clothings”, “冬装” winter clothings. “平均值”-“average value”, “搜索指数”-“search indexes”.

As Fig 2 is also the screenshot from the Baidu website, there are Chinese words in the figure. We list the Chinese words in the Fig.2 from left to right, and top to down. where “整体趋势”means “whole trend”, “PC 趋势”-“PC trend”, “移动趋势” “mobile trend”, “最近”-“recent”, “7 天”-“7 days”, “30 天”-“30days”, “90 天”-“90 days”, “半年”-“half a year”, “全部”-“all”, “自定义”-“custom”,“平均值”-“average value”, “搜索指数”-“search indexes”.

As shown in Fig. 2, the access frequency of work-related words has the same tidal periodic access characteristics: the peak points usually appear on workdays and the valley points almost always appear on weekends. This kind of weekly tidal period phenomenon always appears in work-related words, documents and media.

As Fig3 is also the screenshot from the Baidu website, there are Chinese words in the figure. We list the Chinese words in the Fig.s from left to right, and top to down. where “整体趋势”means “whole trend”, “PC 趋势”-“PC trend”, “移动趋势” “mobile trend”, “最近”-“recent”, “7 天”-“7 days”, “30 天”-“30days”, “90 天”-“90 days”, “半年”-“half a year”, “全部”-“all”, “自定义”-“custom”, “电视剧”-“TV”, “电影”-“movie”, “游戏”-“'game”,“平均值”-“average value”, “搜索指数”-“search indexes”.

On the other hand, as shown in Fig. 3, the access frequency of entertainment-related words also has the same tidal periodic access characteristics. The peak points usually appear on weekends, and the valley points almost always appear on workdays. This kind of weekly tidal period phenomenon always appears in entertainment-related words, documents and media.

Recently, placing data according to the access characteristics is one of the main techniques for energy saving in large scale storage systems [8] [11]. However, only the temporal data access characteristics are considered in the existing literatures.

Based on the above observations and due to a lack of consideration of the periodic data access characteristics of the current existing energy-aware data classification work, we focused on extracting the seasonal and tidal access characteristics for energy-aware data clustering storage in this paper. In this direction, our paper makes the following key contributions:

(1) Data seasonal and tidal access characteristics extracting algorithms (SCEA and TCEA) are designed, in which how to store the data access frequency and how to express the seasonal and tidal characteristics are described in detail.

(2) The energy-aware data clustering strategy K-ear is designed, in which the framework of K-ear is first constructed and then the data are clustered by an unsupervised machine learning algorithm into different categories. Correspondingly, the cloud storage system is divided into fifteen zones to store data with similar access characteristics. 
(3) The proposed K-ear algorithm is modeled, and the classical data classification SEA and the Hadoop default data placement algorithms are used for comparison through a mathematical method. The constructed mathematics model is the basis for analyzing the energy efficiency of the proposed K-ear strategy and is also used to conduct simulation experiments to verify the advantage of the proposed strategy in energy consumption.

(4) Substantial simulation experiments are conducted using extended CloudSimDisk simulator, and the results demonstrate that the proposed K-ear strategy is more energy-efficient than the other two data placement algorithms.

The rest of the paper is organized as follows. In Section 2, we analyze the related energy-aware data classification work. The detailed framework and the algorithms of K-ear are described in Section 3. The mathematical model of the proposed K-ear strategy and the classical energy-aware data classification algorithm SEA are constructed for energy and performance analysis in Section 4. In Section 5, we demonstrate the detailed energy consumption and performance of the proposed K-ear, SEA and Hadoop default data storage algorithms. Conclusions and future work are presented in the final section.

\section{Related Work}

Energy-aware data classification strategies classify data into different categories according to the data access characteristics and then partition the storage system into different zones. Data with similar access characteristic clustering are stored in the same storage zone. Different zones run in different energy and performance modes. Energy consumption savings are achieved by managing the power state of the different zones. T. Xie first proposed a striping-based energy-aware data placement strategy (SEA) in the RAID (Redundant Arrays of Independent Disks) storage system [12], in which RAID disks are divided into Hot Disk Zone and Cold Disk Zone. Popular data are stored in the Hot Disk Zone while unpopular data are stored in the Cold Disk Zone. Disks in Hot Disk Zone run in a mode with a high transfer rate and high power rate, while disks in Cold Disk Zone run in a mode with a low transfer rate and low power rate. Analysis and simulation results show that the proposed SEA mechanism saves much energy consumption with little performance loss. Analyzing the access traces in Yahoo showed that the data access patterns in the Hadoop cluster have obvious heterogeneity. R.T. Kaushik et al. designed the GreenHDFS mechanism, in which data are classified into different categories according to temperature. The temperature of data is deduced by their availability and the user's performance requirement. Correspondingly, the Hadoop cluster is divided into Hot Zone and Cold Zone. Simulation experiments conducted on the generated workload based on a real trace of three months of data from Yahoo demonstrate that $26 \%$ of energy consumption can be reduced by only managing the cold zone at a lower power consumption rate while the system load is light [13]. A similar energy-aware data classification policy, Lightning, was designed by the Kaushik team [14] to reduce the energy consumption of the Yahoo cloud storage system. Inspired by GreenHDFS and the lighting mechanism, we have proposed a green data classification strategy based on anticipation named AGDC, in which the neural network is employed to predict the temperature of data. Based on the predicted temperature of data, they are classified into three categories: cold data, seasonal hot data and hot data. Correspondingly, the cloud storage system is divided into different zones. Simulation experiments conducted on the GridSim simulator demonstrated that the AGDC mechanism can save approximately 16\% of energy consumption at the expense of an increased average response time of $0.005 \mathrm{~s}$. AGDC outperformed the integration general classification algorithm TDCS [15]. In the literature [19], data are classified into different categories according to their access frequency and regularity. RACK is divided into Active-Zone and Sleep-Zone. Data with different access characteristics are stored in different zones. Simulation experimental results in the MATLAB and GridMix environments show that the energy consumption saved by the proposed algorithm is up to $39.01 \%$. An energy-efficient algorithm that classifies data in the cloud storage system was proposed by Z. Tao et al. [16] that divided the cloud storage system into HotZone, ColdZone and Reduplication Zone. Data are stored in the zones according to their repetition and activity factor characteristics. The experimental 
results show that the energy utilization rate improved by $25 \%$. Furthermore, the proposed algorithm performs better when the system load is light. Recently, the SLA (Service Level Agreement) has been considered in more and more literatures for trading off the energy efficiency and the QoS. A dynamic data aggregation algorithm for green cloud computing is proposed in [17]. According to the data access pattern, the data are aggregately stored dynamically among nodes. By managing the power state of the storage nodes, energy consumption can be reduced along with the QoS considered. Dr. Long designed static and dynamic file layout and replica and data layout policies to reduce the energy consumption in cloud storage systems [18]. The static file layout strategy (SFLS) first divides data into hot files and big files according to their access frequency and service time. Correspondingly, the disks are divided into different groups. I/O requests are distributed to the different disk groups according to the access frequency and service time. The results obtained from the CloudSim simulator demonstrate that SFLS can save power consumption by over 35\% compared to the default HDFS. Moreover, R.Yadav et la published the related article for minimizing energy consumption and SLA violation in cloud computing[23] [26]. Other data placement or data layout strategies for energy efficiency have been published in recent years [19] [35], but the period access characteristics also have not been extracted for data clustering storing.

As described before, data classification is an efficient way to reduce energy consumption. However, only the instant data access frequency is considered in all of the above energy-aware data classification strategies, which may cause frequent data migration and result in performance loss. The seasonal and tidal periodic access characteristics of data are thoroughly extracted for data classification storing in our proposed K-ear strategy, which can leave the nodes in a low power state for a relatively long time, leading to energy consumption savings.

A summary of the reviewed related work is presented in Table 1, comparing in terms of whether the access frequency or the periodic access characteristics (seasonal or tidal periodic access characteristics) has been considered or not, the zones have been divided.

\section{K-ear: Energy-aware Data Clustering Strategy}

The proposed K-ear strategy consists of two data access characteristics extraction algorithms (Seasonal Characteristics Extracting Algorithm SCEA, and Tidal Characteristics Extracting Algorithm TCEA) and a framework, which are described in the following subsections.

\subsection{Periodic Data Access Characteristics Extraction}

The data seasonal and tidal access characteristics are extracted by the SCEA and TCEA algorithms, respectively. To explain the SCEA and TCEA algorithms clearly, some definitions are given first.

Representation Data Set: $D=\left\{d_{1}, d_{2}, \cdots, d_{m}\right\}$, which is the representation data set of the primary data for clustering, and $m$ is the number of data.

Data Seasonal Characteristics: are represented by the vector $\mathrm{SE}=\left[\begin{array}{c}\mathrm{Se}_{1} \\ \mathrm{Se}_{2} \\ \vdots \\ \mathrm{Se}_{m}\end{array}\right]$. Assume that $\mathrm{y}$ is the number of years of data to be collected and that there are four seasonal search index ratios for each year. Therefore, $\mathrm{Se}_{i}=\left[s e_{1}, s e_{2}, \cdots, s e_{4 * y}\right]$.

Data Tidal Characteristics: are represented by the vector $C X=\left[\begin{array}{c}c x_{1} \\ c x_{2} \\ \vdots \\ c x_{m}\end{array}\right]$ and $c x_{i}=\left[\begin{array}{lllllll}\mathrm{p}_{\mathrm{i}, 1} & \mathrm{v}_{\mathrm{i}, 1} & \mathrm{p}_{\mathrm{i}, 2} & \mathrm{v}_{\mathrm{i}, 2} & \cdots & \mathrm{p}_{\mathrm{i}, \mathrm{z}} & \mathrm{v}_{\mathrm{i}, \mathrm{z}}\end{array}\right]$, in which $\mathrm{z}$ is the number of weeks of representation data (there are 52 weeks in one year).

The seasonal characteristics extracting algorithm is described in Algorithm 1, through which the seasonal characteristics of the data stored in the SE vector. 
Tidal characteristics extracting algorithm is described in Algorithm 2, through which the seasonal characteristics of the data are stored in the $\mathrm{CX}$ vector..

\subsection{Framework and Strategy of K-ear}

The main procedures of the proposed K-ear are delineated in Fig. 4. The seasonal access characteristics of data are extracted by the SCEA algorithm (see Algorithm 1 for details). Then, the machine learning clustering-related algorithm is employed on the extracted seasonal access characteristics. Data are clustered into five categories: spring data, summer data, autumn data, winter data and other data. Correspondingly, the cloud storage system is divided into five zones: the spring zone, summer zone, autumn zone, winter zone and other zones. The different data categories are stored in the corresponding storage zones. When entering a certain zone, the TCEA (Algorithm 2 for detail) is utilized to extract the tidal access characteristics of data. Similarly, the machine learning clustering-related algorithm is employed on the extracted tidal access characteristics. The data are further clustered into three categories: work-related data, entertainment data and other data. Therefore, the cloud storage system is partitioned into fifteen zones: the spring-work zone, spring-entertainment zone, spring-other zone, summer-work zone, summer-entertainment zone, summer-other zone, autumn-work zone, autumn-entertainment zone, autumn-other zone, winter-work zone, winter-entertainment zone, winter-other zone, other-work zone, other-entertainment zone, and other-other zone. We assume that a disk has two speed modes: a high-speed mode with a high transfer rate and a high energy-consuming rate and a low-speed mode with a low transfer rate and a low energy consumption rate, as it is a common method for modeling hard-disk power consumption [12][38][39][40]. According to the different periods of the year, different storage zones run in different speed modes. For example, on a workday in the spring, the spring-work zone, other-work zone and other-other zone run in the high speed mode for performance consideration. The other zones run in low-speed mode to save energy consumption.

The symbols used in the proposed K-ear algorithm are explained in Table 2 and Table3

When the $s$ in the notation $\eta_{s}$ is replaced respectively by $m$, a, and $w$, notations meaning the related characteristics of the Summer, Autumn and Winter respectively.

And the above symbols have the following relationships: $\eta_{s}+\eta_{m}+\eta_{a}+\eta_{w}+\eta_{o}=1, \eta_{s-\mathrm{w}}+\eta_{s-\mathrm{h}}+\eta_{s-\mathrm{o}}=$ $1, \eta_{m-\mathrm{w}}+\eta_{m-\mathrm{h}}+\eta_{m-\mathrm{o}}=1, \eta_{a-\mathrm{w}}+\eta_{a-\mathrm{h}}+\eta_{a-\mathrm{o}}=1, \eta_{w-\mathrm{w}}+\eta_{w-\mathrm{h}}+\eta_{w-\mathrm{o}}=1, \eta_{o-\mathrm{w}}+\eta_{o-\mathrm{h}}+\eta_{o-\mathrm{o}}=1$.

Assume the number of the disks is $n$, and the disks set is defined as below. $\mathrm{K}=$ $\left\{\mathrm{s}_{1}, \mathrm{~s}_{2}, \cdots, \mathrm{s}_{n 1}, \mathrm{~m}_{1}, \mathrm{~m}_{2}, \cdots \mathrm{m}_{n 2}, \mathrm{a}_{1}, \mathrm{a}_{2}, \cdots \mathrm{a}_{n 3}, w_{1}, w_{2}, \cdots, w_{n 4}, \mathrm{o}_{1}, \mathrm{o}_{2}, \cdots \mathrm{o}_{n 5}\right\}$

And the set $\mathrm{S}=\left\{\mathrm{s}_{1}, \mathrm{~s}_{2}, \cdots, \mathrm{s}_{n 1}\right\} \quad M=\left\{\mathrm{m}_{1}, \mathrm{~m}_{2}, \cdots \mathrm{m}_{n 2}\right\} \quad A=\left\{\mathrm{a}_{1}, \mathrm{a}_{2}, \cdots \mathrm{a}_{n 3}\right\} \quad W=\left\{w_{1}, w_{2}, \cdots, w_{n 4}\right\} \quad O=$ $\left\{\mathrm{o}_{1}, \mathrm{o}_{2}, \cdots \mathrm{o}_{n 5}\right\}$ represent the disks store the data with spring, summer, autumn, winter and no seasonal characteristics respectively, in which $\mathrm{n}_{1}=\eta_{s} \times n, \mathrm{n}_{2}=\eta_{m} \times n, \mathrm{n}_{3}=\eta_{a} \times n, \mathrm{n}_{4}=\eta_{w} \times n, \mathrm{n}_{5}=\eta_{o} \times n$. And the number of the disks to store the data with tidal characteristics of every seasonal zone are listed in Table 2 .

In Table 3, when the $s$ in the notation $\eta_{s}$ is respectively replaced by $\mathrm{m}$, a, and $\mathrm{w}, \& \& n_{1}$ is respectively replcaed by $n_{2}, n_{3}, n_{4}$ notations meaning the number of the disk to store the data with related characteristics of the Summer, Autumn and Winter. Respectively.

The detailed procedures of the K-ear strategy are illustrated in Algorithm 3.

According to the algorithm 3, the number of disks running in different modes during different time zones are listed in Table 4.

\section{Mathematic Modeling}

To analyze the energy efficiency advantage of our proposed K-ear strategy, we model the K-ear mathematically in this section. The parameters used during mathematical modeling are listed in following tables. Parameters of the whole system are 
listed in Table5. Parameters about the spring season are listed in Table 6. Parameters about the summer season, autumn season and winter season will be explained correspondingly.

For short, when the spring in the labels of the Table 6 is replaced by the summer, autumn and winter respectively, the parameters are about the Summer season, Autumn season and Winter season respectively. Such as spring_work_t $t_{h}^{\text {active }}$ means the total time of disks running in high mode with active status during workdays in Spring, and the summer_work_tactive means the total time of disks running in high mode with active status during workdays in Summer. Furthermore, when the $S$ in the labels of the Table 6 is replaced by the $M, A, W$ respectively, the parameters are about the Summer season, Autumn season and Winter season respectively. Such as $S_{-} W_{h}^{n}$ means the total access times in high mode during workdays in Spring, and the $M_{-} W_{h}^{n}$ means the total access times in high mode during workdays in Summer. The replacement rule is the same for the remaining parameters.

According to the proposed K-ear strategy in Algorithm 3, the energy consumption model of K-ear is deduced as below.

$\mathrm{e}_{\text {total }}=$ spring_work_ $\mathrm{e}_{\text {total }}+$ spring_holiday_ $\mathrm{e}_{\text {total }}+$ summer_work_e $\mathrm{e}_{\text {total }}+$

+ summer_holiday_e $\mathrm{e}_{\text {total }}+$ autumn_work_e $\mathrm{e}_{\text {total }}+$ autumn_holiday_e $\mathrm{e}_{\text {total }}+$ winter_work_e $\mathrm{e}_{\text {total }}$

+winter_holiday_e $\mathrm{e}_{\text {total }}$

The intuition behind the equations (1) is that the total energy consumption of the systems is the summary of the energy consumption of the workdays in spring (spring_work_e $\mathrm{e}_{\text {total }}$ ), holidays in spring (spring_holiday_e $\mathrm{e}_{\text {total }}$ ), workdays in summer (summer_work_e $\mathrm{e}_{\text {total }}$ ), holidays in summer (summer_holiday_e $\mathrm{t}_{\text {total }}$ ), workdays in autumn $\left(\right.$ autumn_work_e $\left.\mathrm{e}_{\text {total }}\right)$, holidays in autumn (autumn_holiday_e $\left.\mathrm{e}_{\text {total }}\right)$, workdays in winter (winter_work_e $\left.\mathrm{e}_{\text {total }}\right)$, holidays in winter (winter_holiday_e $\mathrm{e}_{\text {total }}$ ). And there are calculated by the following formulas

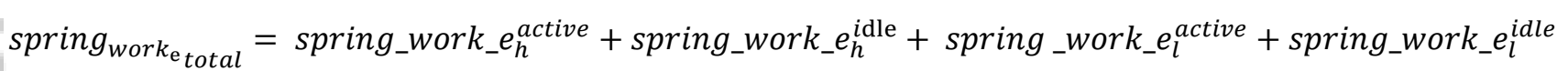

(2)

summer_work_ $\mathrm{e}_{\text {total }}=$ summer_work_ $e_{h}^{\text {active }}+$ summer_work_ $e_{h}^{\text {idle }}+$ summer_work_ $e_{l}^{\text {active }}+$ summer_work_e $e_{l}^{i d l e}$

autumn_work_e $\mathrm{e}_{\text {total }}=a u t u m n \_w o r k \_e_{h}^{\text {active }}+a u t u m n \_w o r k \_e_{h}^{i d l e}+a u t u m n \_w o r k \_e_{l}^{a c t i v e}+$ autumn_work_e $e_{l}^{\text {idle }}$

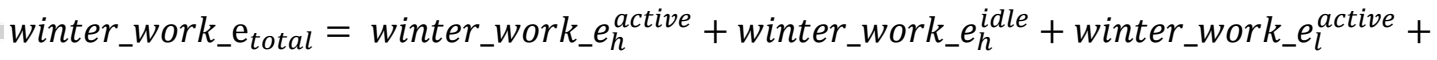
winter_work_e $e_{l}^{i d l e}$

spring_holiday_e $e_{t o t a l}=$ spring_holiday_ $e_{h}^{\text {active }}+\operatorname{spring}_{-}$holiday_e $e_{h}^{i \mathrm{dle}}+\operatorname{spring}_{-}$holiday_$e_{l}^{\text {active }}+$ spring_holiday_e $e_{l}^{i d l e}$

summer_holiday_e $\mathrm{e}_{\text {total }}=$ summer_holiday_e $e_{h}^{\text {active }}+$ summer_holiday_$_{h} e_{h}^{\text {idle }}+$ summer_holiday_e $e_{l}^{\text {active }}+$ summer_holiday_e $e_{l}^{i d l e}$

autumn_holiday_e $\mathrm{e}_{\text {total }}=$ autumn_holiday_e $e_{h}^{\text {active }}+$ autumn_holiday_e $e_{h}^{\text {idle }}+$ autumn_holiday_e $e_{l}^{\text {active }}+$ autumn_holiday_e $e_{l}^{i d l e}$

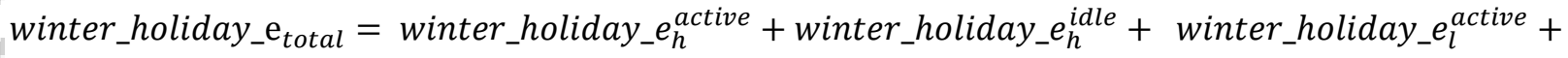
winter_holiday_e $e_{l}^{i d l e}$

When

spring_work_ $e_{h}^{\text {active }}=\mathrm{p}^{h} \times$ spring_work_t $t_{h}^{\text {active }}=\mathrm{p}^{h} \times S_{-} W_{h}^{n} \times \mathrm{s}^{\prime} /\left(\tau^{h} \times H 1\right)$ 
spring_work_ $e_{h}^{i d l e}=i^{h} \times\left(\right.$ spring_work_T $\times H 1-$ spring_work_t $\left.t_{h}^{\text {active }}\right)=i^{h} \times\left(\frac{5 T}{28} \times H 1-\right.$

$S_{-} W_{h}^{n} \times \mathrm{s}^{\prime} /\left(\tau^{h} \times H 1\right)$

spring_holiday_e $\boldsymbol{e}_{h}^{\text {active }}=\mathrm{p}^{h} \times$ spring_holiday_t $t_{h}^{\text {active }}=\mathrm{p}^{h} \times S_{-} H_{h}^{h} \times \mathrm{s}^{\prime} /\left(\tau^{h} \times H 2\right)$

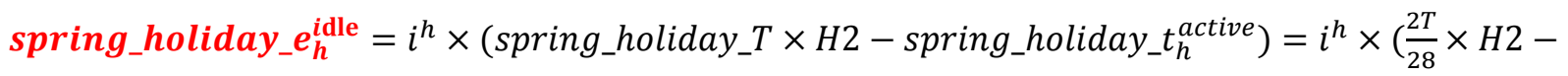

$\left.S_{H h}^{n} \times \mathrm{s}^{\prime} /\left(\tau^{h} \times H 2\right)\right)$

And the other formulas to calculate the valuses related to the Summer season, Autumn season and Winter season are simluar to the formulas from (10) to (13), as they accorrding to the above replacement rules plus that $\mathrm{H} 1$ and $\mathrm{H} 2$ are replcaed by $\mathrm{H} 3$ and $\mathrm{H} 4, \mathrm{H} 5$ and $\mathrm{H} 6, \mathrm{H} 7$ and $\mathrm{H8}$.

spring_work $\boldsymbol{e}_{l}^{\text {active }}=\mathrm{p}^{l} \times$ spring_work_t $t_{l}^{\text {active }}=\mathrm{p}^{l} \times S_{-} W_{l}^{n} \times \mathrm{s}^{\prime} /\left(\tau^{l} \times L 1\right)$

spring_work $\boldsymbol{e}_{l}^{i \mathrm{dle}}=i^{l} \times\left(\right.$ spring_work_$T \times L 1-$ spring_work $\left._{-} t_{l}^{\text {active }}\right)=i^{l} \times\left(\frac{5 T}{28} \times L 1-\right.$

$\left.S_{-} W_{l}^{n} \times \mathrm{s}^{\prime} /\left(\tau^{l} \times L 1\right)\right)$

spring_holiday_e $\boldsymbol{e}_{l}^{\text {active }}=\mathrm{p}^{l} \times$ spring_holiday $_{-} t_{l}^{\text {active }}=\mathrm{p}^{l} \times S_{-} H_{l}^{n} \times \mathrm{s}^{\prime} /\left(\tau^{l} \times L 2\right)$

spring_holiday_ $e_{l}^{i d l e}=i^{l} \times\left(\right.$ spring_holiday_$_{-} \times L 2-$ spring_holiday_t $\left._{l}^{\text {active }}\right)=i^{l} \times\left(\frac{2 T}{28} \times L 2-\right.$

$\left.S_{-} H_{l}^{n} \times \mathrm{s}^{\prime} /\left(\tau^{l} \times L 2\right)\right)$

And the other formulas to calculate the valuses related to the Summer season, Autumn season and Winter season are simluar to the formulas from (14) to (17), as they accorrding to the above replacement rules plus that L1 and L2 are replcaed by L3 and L4, L5 and L6, L7 and L8.

Therefore,

$$
\begin{array}{r}
\mathrm{e}_{\text {total }}=\mathrm{p}^{h} \times S_{-} W_{h}^{n} \times \mathrm{s}^{\prime} /\left(\tau^{h} \times H 1\right)+i^{h} \times\left(\frac{5 T}{28} \times H 1-S_{-} W_{h}^{n} \times \mathrm{s}^{\prime} /\left(\tau^{h} \times H 1\right)+\mathrm{p}^{h} \times S_{-} H_{h}^{n} \times \mathrm{s}^{\prime} /\left(\tau^{h} \times H 2\right)+i^{h} \times\right. \\
\left(\frac{2 T}{28} \times H 2-S_{-} H_{h}^{n} \times \mathrm{s}^{\prime} /\left(\tau^{h} \times H 2\right)+\mathrm{p}^{h} \times M_{-} W_{h}^{n} \times \mathrm{s}^{\prime} /\left(\tau^{h} \times H 3\right)+i^{h} \times\left(\frac{5 T}{28} \times H 3-M_{-} W_{h}^{n} \times \mathrm{s}^{\prime} /\left(\tau^{h} \times H 3\right)+\mathrm{p}^{h} \times\right.\right. \\
M_{-} H_{h}^{n} \times \mathrm{s}^{\prime} /\left(\tau^{h} \times H 4\right)+i^{h} \times\left(\frac{2 T}{28} \times H 4-M_{-} H_{h}^{n} \times \mathrm{s}^{\prime} /\left(\tau^{h} \times H 4\right)+\mathrm{p}^{h} \times A_{-} W_{h}^{n} \times \mathrm{s}^{\prime} /\left(\tau^{h} \times H 5\right)+i^{h} \times\left(\frac{5 T}{28} \times H 5-\right.\right. \\
A_{-} W_{h}^{n} \times \mathrm{s}^{\prime} /\left(\tau^{h} \times H 5\right)+\mathrm{p}^{h} \times A_{-} H_{h}^{n} \times \mathrm{s}^{\prime} /\left(\tau^{h} \times H 6\right)+i^{h} \times\left(\frac{2 T}{28} \times H 6-A_{-} H_{h}^{n} \times \mathrm{s}^{\prime} /\left(\tau^{h} \times H 6\right)+\mathrm{p}^{h} \times\right. \\
W_{-} W_{h}^{n} \times \mathrm{s}^{\prime} /\left(\tau^{h} \times H 7\right)+i^{h} \times\left(\frac{5 T}{28} \times H 7-W_{-} W_{h}^{n} \times \mathrm{s}^{\prime} /\left(\tau^{h} \times H 7\right)+\mathrm{p}^{h} \times W_{-} H_{h}^{n} \times \mathrm{s}^{\prime} /\left(\tau^{h} \times H 8\right)+i^{h} \times\left(\frac{2 T}{28} \times\right.\right. \\
H 8-W_{-} H_{h}^{n} \times \mathrm{s}^{\prime} /\left(\tau^{h} \times H 8\right)+\mathrm{p}^{l} \times S_{-} W_{l}^{n} \times \mathrm{s}^{\prime} /\left(\tau^{l} \times L 1\right)+i^{l} \times\left(\frac{5 T}{28} \times L 1-S_{-} W_{l}^{n} \times \mathrm{s}^{\prime} /\left(\tau^{l} \times L 1\right)\right)+\mathrm{p}^{l} \times \\
S_{-} H_{l}^{n} \times \mathrm{s}^{\prime} /\left(\tau^{l} \times L 2\right)+i^{l} \times\left(\frac{2 T}{28} \times L 2-S_{-} H_{l}^{n} \times \mathrm{s}^{\prime} /\left(\tau^{l} \times L 2\right)\right)+\mathrm{p}^{l} \times M_{-} W_{l}^{n} \times \mathrm{s}^{\prime} /\left(\tau^{l} \times L 3\right)+i^{l} \times\left(\frac{5 T}{28} \times L 3-\right.
\end{array}
$$

This article is protected by copyright. All rights reserved. 
$\left.M_{-} W_{l}^{n} \times \mathrm{s}^{\prime} /\left(\tau^{l} \times L 3\right)\right)+\mathrm{p}^{l} \times M_{-} H_{l}^{n} \times \mathrm{s}^{\prime} /\left(\tau^{l} \times L 4\right)+i^{l} \times\left(\frac{2 T}{28} \times L 4-M_{-} H_{l}^{n} \times \mathrm{s}^{\prime} /\left(\tau^{l} \times L 4\right)\right)+\mathrm{p}^{l} \times$ $A_{-} W_{l}^{n} \times \mathrm{s}^{\prime} /\left(\tau^{l} \times L 5\right)+i^{l} \times\left(\frac{5 T}{28} \times L 5-A_{-} W_{l}^{n} \times \mathrm{s}^{\prime} /\left(\tau^{l} \times L 5\right)\right)+\mathrm{p}^{l} \times A_{-} H_{l}^{n} \times \mathrm{s}^{\prime} /\left(\tau^{l} \times L 6\right)+i^{l} \times\left(\frac{2 T}{28} \times L 6-\right.$ $\left.A_{-} H_{l}^{n} \times \mathrm{s}^{\prime} /\left(\tau^{l} \times L 6\right)\right)+\mathrm{p}^{l} \times W_{-} W_{l}^{n} \times \mathrm{s}^{\prime} /\left(\tau^{l} \times L 7\right)+i^{l} \times\left(\frac{5 T}{28} \times L 7-W_{-} W_{l}^{n} \times \mathrm{s}^{\prime} /\left(\tau^{l} \times L 7\right)\right)+\mathrm{p}^{l} \times$ $W_{-} H_{l}^{n} \times \mathrm{s}^{\prime} /\left(\tau^{l} \times L 8\right)+i^{l} \times\left(\frac{2 T}{28} \times L 8-W_{-} H_{l}^{n} \times \mathrm{s}^{\prime} /\left(\tau^{l} \times L 8\right)\right)(18)$

Assume $\mathrm{B}=\mathrm{p}^{h} \times \mathrm{s}^{\prime} / \tau^{h} \quad \mathrm{C}=\mathrm{p}^{l} \times \times \mathrm{s}^{\prime} / \tau^{l}$

The Energy Consumption Model can be simplified as :

$\mathrm{e}_{\text {total }}=\left(S_{W_{h}}^{n} / H 1+S_{H h}^{n} / H 2+M_{W_{h}^{n}}^{n} / H 3+M_{H h}^{n} / H 4+A_{W_{h}^{n}}^{n} / H 5+A_{H h}^{n} / H 6+W_{W_{h}^{n}}^{n} / H 7+W_{H h}^{n} / H 8\right) \times B+i^{h} \times$ $\left(\frac{5 T}{28} \times H 1-S_{-} W_{h}^{n} \times \mathrm{s}^{\prime} /\left(\tau^{h} \times H 1\right)+i^{h} \times\left(\frac{2 T}{28} \times H 2-S_{-} H_{h}^{n} \times \mathrm{s}^{\prime} /\left(\tau^{h} \times H 2\right)+i^{h} \times\left(\frac{5 T}{28} \times H 3-\right.\right.\right.$ $M_{-} W_{h}^{n} \times \mathrm{s}^{\prime} /\left(\tau^{h} \times H 3\right)+i^{h} \times\left(\frac{2 T}{28} \times H 4-M_{-} H_{h}^{n} \times \mathrm{s}^{\prime} /\left(\tau^{h} \times H 4\right)+i^{h} \times\left(\frac{5 T}{28} \times H 5-A_{-} W_{h}^{n} \times \mathrm{s}^{\prime} /\left(\tau^{h} \times H 5\right)+\right.\right.$ $i^{h} \times\left(\frac{2 T}{28} \times H 6-A_{-} H_{h}^{n} \times \mathrm{s}^{\prime} /\left(\tau^{h} \times H 6\right)+i^{h} \times\left(\frac{5 T}{28} \times H 7-W_{-} W_{h}^{n} \times \mathrm{s}^{\prime} /\left(\tau^{h} \times H 7\right)+i^{h} \times\left(\frac{2 T}{28} \times H 8-\right.\right.\right.$ $W_{-} H_{h}^{n} \times \mathrm{s}^{\prime} /\left(\tau^{h} \times H 8\right)+\left(S_{W_{l}}^{n} / L 1+S_{H_{l}}^{n} / L 2+M_{W_{l}}^{n} / L 3+M_{H_{l}}^{n} / L 4+A_{W_{l}}^{n} / L 5+A_{H_{l}}^{n} / L 6+W_{W_{l}}^{n} / L 7+\right.$ $\left.W_{H_{l}}^{n} / L 8\right) \times C+i^{l} \times\left(\frac{5 T}{28} \times L 1-S_{-} W_{l}^{n} \times \mathrm{s}^{\prime} /\left(\tau^{l} \times L 1\right)\right)+i^{l} \times\left(\frac{2 T}{28} \times L 2-S_{-} H_{l}^{n} \times \mathrm{s}^{\prime} /\left(\tau^{l} \times L 2\right)\right)+i^{l} \times\left(\frac{5 T}{28} \times L 3-\right.$ $\left.M_{-} W_{l}^{n} \times \mathrm{s}^{\prime} /\left(\tau^{l} \times L 3\right)\right)+i^{l} \times\left(\frac{2 T}{28} \times L 4-M_{-} H_{l}^{n} \times \mathrm{s}^{\prime} /\left(\tau^{l} \times L 4\right)\right)+i^{l} \times\left(\frac{5 T}{28} \times L 5-A_{-} W_{l}^{n} \times \mathrm{s}^{\prime} /\left(\tau^{l} \times L 5\right)\right)+i^{l} \times$ $\left(\frac{2 T}{28} \times L 6-A_{-} H_{l}^{n} \times \mathrm{s}^{\prime} /\left(\tau^{l} \times L 6\right)\right)+i^{l} \times\left(\frac{5 T}{28} \times L 7-W_{-} W_{l}^{n} \times \mathrm{s}^{\prime} /\left(\tau^{l} \times L 7\right)\right)+i^{l} \times\left(\frac{2 T}{28} \times L 8-\right.$ $\left.W_{-} H_{l}^{n} \times \mathrm{s}^{\prime} /\left(\tau^{l} \times L 8\right)\right)$

Correspondingly, the energy consumption model of the Hadoop default data placement strategy is as follows, which is without classification:

$$
\mathrm{e}_{\text {total }}^{\prime}={\mathrm{e}^{\prime}}_{h}^{\text {active }}+e_{h}^{\prime \text { idle }}
$$

$\mathrm{e}_{h}^{\prime}{ }_{h}^{\text {active }}=\mathrm{p}^{h} \times\left(S_{W_{h}}^{n}+S_{H_{h}}^{n}+M_{W_{h}}^{n}+M_{H_{h}}^{n}+A_{W_{h}}^{n}+A_{H_{h}}^{n}+W_{W_{h}}^{n}+W_{H_{h}}^{n}+S_{-} W_{l}^{n}+S_{-} H_{l}^{n}+M_{-} W_{l}^{n}+M_{-} H_{l}^{n}\right.$

$$
\left.+A_{-} W_{l}^{n}+A_{-} H_{l}^{n}+W_{-} W_{l}^{n}+W_{-} H_{l}^{n}\right) \times \mathrm{s}^{\prime} /\left(\tau^{h} \times n\right)
$$

$=\left(S_{W_{h}}^{n}+S_{H_{h}}^{n}+M_{W_{h}}^{n}+M_{H_{h}}^{n}+A_{W_{h}}^{n}+A_{H_{h}}^{n}+W_{W_{h}}^{n}+W_{H_{h}}^{n}+S_{W_{l}}^{n}+S_{H_{l}}^{n}+M_{W_{l}}^{n}+M_{H_{l}}^{n}+A_{W_{l}}^{n}+A_{H_{l}}^{n}+W_{W_{l}}^{n}+\right.$ $\left.W_{H l}^{n}\right) \times B / n$

$e_{h}^{\prime \text { idle }}=i^{h} \times\left(T \times n-\left(S_{W_{h}}^{n}+S_{H_{h}}^{n}+M_{W_{h}}^{n}+M_{H_{h}}^{n}+A_{W_{h}}^{n}+A_{H_{h}}^{n}+W_{W_{h}}^{n}+W_{H_{h}}^{n}+S_{-} W_{l}^{n}+S_{-} H_{l}^{n}+M_{-} W_{l}^{n}+M_{-} H_{l}^{n}\right.\right.$

$$
\left.+A_{-} W_{l}^{n}+A_{-} H_{l}^{n}+W_{-} W_{l}^{n}+W_{-} H_{l}^{n}\right) \times \mathrm{s}^{\prime} /\left(\tau^{h} \times n\right)
$$

Therefore, $\mathrm{e}_{\text {total }}^{\prime}=\left(S_{W_{h}}^{n}+S_{H_{h}}^{n}+M_{W_{h}}^{n}+M_{H_{h}}^{n}+A_{W_{h}}^{n}+A_{H h}^{n}+W_{W_{h}}^{n}+W_{H_{h}}^{n}+S_{W_{l}}^{n}+S_{H_{l}}^{n}+M_{W_{l}}^{n}+M_{H_{l}}^{n}+\right.$ $\left.A_{W_{l}}{ }^{n}+A_{H l}{ }^{n}+W_{W_{l}}{ }^{n}+W_{H l}{ }^{n}\right) \times B / n+i^{h} \times\left(T \times n-\left(S_{W_{h}}^{n}+S_{H h}^{n}+M_{W_{h}}{ }^{n}+M_{H h}{ }^{n}+A_{W_{h}}{ }^{n}+A_{H h}{ }^{n}+W_{W_{h}}^{n}+\right.\right.$ $\left.W_{H h}^{n}+S_{-} W_{l}^{n}+S_{-} H_{l}^{n}+M_{-} W_{l}^{n}+M_{-} H_{l}^{n}+A_{-} W_{l}^{n}+A_{-} H_{l}^{n}+W_{-} W_{l}^{n}+W_{-} H_{l}^{n}\right) \times \mathrm{s}^{\prime} /\left(\tau^{h} \times n\right)$ 
And the Energy Consumption model of SEA classification [12] can be deduced by the following formula:

$e_{\text {total }_{\text {sea }}}=e_{\text {hot }}+e_{\text {cold }}=e_{\text {hot }}^{\text {active }}+e_{\text {hot }}^{\text {idle }}+e_{\text {cold }}^{\text {active }}+e_{\text {cold }}^{\text {idle }}=\mathrm{p}^{h} \times n_{h} \times \mathrm{s}^{\prime} /\left(\tau^{h} \times \eta_{h} \times n\right)+i^{h} \times$

$\left(T \times \eta_{h} \times n-n_{h} \times \mathrm{s}^{\prime} /\left(\tau^{h} \times \eta_{h} \times n\right)\right)+\mathrm{p}^{l} \times n_{c} \times \mathrm{s}^{\prime} /\left(\tau^{l} \times \eta_{c} \times n\right)+i^{l} \times\left(T \times \eta_{c} \times n-n_{c} \times \mathrm{s}^{\prime} /\left(\tau^{l} \times \eta_{c} \times n\right)\right)$

(21)

Where $\eta_{h}$ is the ratio of hot (popular) data and $\eta_{c}$ is the ratio of cold (unpopular) data. $n_{h}$ is the total access time of hot data, and $n_{c}$ is the total access time of cold data.

As the mathematical models of the K-ear, Hadoop-default and SEA are stated in formula (19), (20), (21) respectively. We can deduce that the time complexity of the three algorithms depends on the time to calculate the access frequency of the data. That is to say, the time complexity of the three algorithms is $\mathrm{O}(\mathrm{n})$. On the other hand, space complexity of the three algorithms depends on the space to store the access frequency of the data. That is to say, the space complexity of the three algorithms is also O(n). As the analyzed above, the advantage of the three algorithms are usually depends on the energy consumption savings.

Based on the energy consumption model, simulation experiments with the K-ear algorithm and the SEA and Hadoop default algorithms will be conducted in the following section.

\section{Performance Evaluation}

To evaluate the energy efficiency of the proposed K-ear strategy, we generate the workload according to the real access trace from the wiki (wiki workload) and assume a disk with two speed modes. The different transfer rates and energy consumption rates are extracted from article [12]. All of the simulation experiments are conducted in CloudSimDisk [36], which builds on the CloudSim [37] simulator and adds storage simulation capabilities. And the detailed experimental parameters about the software and hardware configuration are listed in Table 7.

The general parameters utilized in the experiments are listed in Table 8.

Disks with two speed modes, the workload and the disk zone, are modeled in the CloudSimDisk environment. The energy efficiency of the three algorithms is evaluated by setting the following ratios: (1) The ratio of the high speed disk utilization to the cloud storage system utilization, (2) the ratio of the data with seasonal characteristics to the data without seasonal characteristics, (3) the ratio of the data with tidal characteristics to the data without tidal characteristics, and (4) the ratio of the hot data to the cold data.

\subsection{The impact of the different ratios of high-speed disk utilization on cloud storage system utilization}

The parameters corresponding to the values set in the experiment are listed in Table 9.

In Table 9, The ratios of the other seasons with the different characteristics are the same as the Spring season, with the $s$ in the notation $\eta_{s}$ is respectively replaced by $\mathrm{m}$, a, and $\mathrm{w}$.

In order to calculate the energy consumption of the SEA algorithm, the ratio of the hot data to cold data is set to 4: 6 in this experiment. Obtained experiment results are demonstrated in the following tables (Table 10, Table 11, Table 12), when the ratios of the high disk utilization to the whole system utilization are 1.6, 1.8, 2.0 respectively.

As shown in the Table 10, the ratio of the high disk utilization to the whole system utilization is 1.6, energy consumed by the K-ear is least, and the most is the Hadoop-default. While the energy consumed by the SEA is fall in between. Compared to the Hadoop-default, the average energy consumption saved by the K-ear is more than $40 \%$, while compared to the SEA, the saved energy consumption is about $11 \%$. Moreover, we have found that the system utilization has little impact on the energy consumption for the three algorithms. 
As shown in the Table 11, when the ratio of the high disk utilization to the whole system utilization is 1.8, the energy consumed by the K-ear is least, and the most is the Hadoop-default. While the energy consumed by the SEA is fall in between. Compared to the Hadoop-default, the average energy consumption saved by the K-ear is about $42 \%$, while compared to the SEA, the saved energy consumption is also about $11 \%$. Moreover, we also have found that the system utilization has little impact on the energy consumption for the three algorithms in this experiment.

As shown in the Table 12, when the ratio of the high disk utilization to the whole system utilization is 2.0, the energy consumed by the K-ear is least, and the most is the Hadoop-default. While the energy consumed by the SEA is fall in between. Compared to the Hadoop-default, the average energy consumption saved by the K-ear is about 42\%, while compared to the SEA, the saved energy consumption is also about $11 \%$. Moreover, we also have found that the system utilization has little impact on the energy consumption for the three algorithms in this experiment.

The above experimental results show that under the different ratios of high-speed disk utilization to system utilization, the K-ear strategy consumes the least energy and the Hadoop default strategy consumes the most energy. The amount of energy consumed by SEA is between the amounts consumed by the K-ear strategy and the Hadoop default algorithm. Moreover, the energy consumption of the cloud storage system only slightly increased as the ratio increased.

\subsection{The impact of the different ratios of data with seasonal characteristics to data without seasonal characteristics}

The ratio of the high-speed disk utilization to the system utilization is set to 2.0, and the ratio of hot data to cold data is set to 4:6 in all of the experiments (experiment 1, experiment 2, and experiment 3 ) in this subsection. The other parameter values are listed in the following tables (Table 13, Table 14).

In Table 13, the ratios of the other seasons with the different characteristics are the same as the Spring season, with the $s$ in the notation $\eta_{s}$ is respectively replaced by $\mathrm{m}$, a, and $\mathrm{w}$.

As the value set as in Table 12 and Table 13, the obtained experimental results are demonstrated in Table 15.

As shown in the Table 15, when the experimental parameters are set as in Table 12 and Table 13, the energy consumed by the K-ear is least, and the most is the Hadoop-default. While the energy consumed by the SEA is also fall in between. Compared to the Hadoop-default, the average energy consumption saved by the K-ear is about 36\%, while compared to the SEA, the saved energy consumption is also about $1.3 \%$, which is almost the same. Moreover, we also have found that the system utilization has little impact on the energy consumption for the three algorithms in this experiment.

The obtained experimental results are demonstrated in Table 17.

As shown in the Table 17, when the experimental parameters are set as in Table 13 and Table 16, the energy consumed by the K-ear is least, and the most is the Hadoop-default. While the energy consumed by the SEA is also fall in between. Compared to the Hadoop-default, the average energy consumption saved by the K-ear is about $40 \%$, while compared to the SEA, the saved energy consumption is also about 7\%. Moreover, we also have found that the system utilization has little impact on the energy consumption for the three algorithms in this experiment.

Seasonal characteristics related parameters' value set in experiment 3 are listed in Table 18

The obtained experimental results are demonstrated in Table 19.

As shown in the Table 19, when the experimental parameters are set as in Table 12 and Table 17, the energy consumed by the SEAis least, and the most is the Hadoop-default. While the energy consumed by the proposed K-ear is fall in between. Compared to the Hadoop-default, the average energy consumption saved by the K-ear is about $32 \%$, while compared to the SEA, 
the energy consumed by K-ear is increased is about $4 \%$, which has little difference. Moreover, we also have found that the system utilization has little impact on the energy consumption for the three algorithms in this experiment.

As shown in the abovementioned experimental results, the K-ear and SEA strategies consume less energy than the Hadoop default strategy under different ratios of data with seasonal characteristics to data without seasonal characteristics. When the data has a higher ratio of seasonal characteristics of 6:4, K-ear slightly outperforms SEA in energy consumption. It can also be found that the lower the ratio of seasonal characteristics (48:52), SEA is more energy-efficient than K-ear but with little difference.

\subsection{The impact of different ratios of data with tidal characteristics to data without tidal characteristics}

The energy consumption of the three strategies with different ratios of data with tidal characteristics is tested in this subsection. The common parameters used in the different experiments are listed in Table 20.

And the tidal characteristics related parameters used in the first experiment are listed in Table 21.

The ratios of the other seasons with the different characteristics are the same as the Spring season, with the $s$ in the notation $\eta_{s}$ is respectively replaced by $\mathrm{m}$, a, and $\mathrm{w}$.

As the parameters of the first experiment set as the above tables, the obtained experimental results are shown in Table 22.

As shown in the Table 22, when the ratio of the data with tidal characteristics to the data without tidal characteristics is 4:6, the energy consumed by the K-ear is least, and the most is the Hadoop-default. While the energy consumed by the SEA is also fall in between. Compared to the Hadoop-default, the average energy consumption saved by the K-ear is about $40 \%$, while compared to the SEA, the saved energy consumption is also about 7\%. Moreover, we also have found that the system utilization has little impact on the energy consumption for the three algorithms.

Tidal characteristics related parameters used in the second experiment are listed in Table 23.

In Table 23, the ratios of the other seasons with the different characteristics are the same as the Spring season, with the $s$ in the notation $\eta_{s}$ is respectively replaced by $\mathrm{m}$, a, and $\mathrm{w}$.

As the parameters of the second experiment set as the above tables, the obtained experimental results are shown in Table 24.

As shown in the Table 24, when the ratio of the data with tidal characteristics to the data without tidal characteristics is 5:5, the energy consumed by the K-ear is least, and the most is the Hadoop-default. While the energy consumed by the SEA is also fall in between. Compared to the Hadoop-default, the average energy consumption saved by the K-ear is about $41 \%$, while compared to the SEA, the saved energy consumption is also about 9\%. Moreover, we also have found that the system utilization has little impact on the energy consumption for the three algorithms in this experiment.

Tidal characteristics related parameters used in the third experiment are listed in Table 25.

In Table 24, the ratios of the other seasons with the different characteristics are the same as the Spring season, with the $s$ in the notation $\eta_{s}$ is respectively replaced by $\mathrm{m}$, a, and $\mathrm{w}$.

As the parameters of the third experiment set as the above tables, the obtained experimental results are shown in Table 26.

As shown in the Table 26, when the ratio of the data with tidal characteristics to the data without tidal characteristics is 2:8, the energy consumed by the K-ear is least, and the most is the Hadoop-default. While the energy consumed by the SEA is also fall in between. Compared to the Hadoop-default, the average energy consumption saved by the K-ear is about 37\%, while compared to the SEA, the saved energy consumption is also about 3.5\%. Moreover, we also have found that the system utilization has little impact on the energy consumption for the three algorithms.

The results of the above three experiments demonstrate that our proposed K-ear strategy is more energy-efficient than the SEA algorithm when the ratio of data with tidal characteristics is high. Furthermore, K-ear has an energy efficiency advantage over the Hadoop default strategy whenever the ratio is high or low. 


\subsection{The impact of the different ratios of hot data to cold data}

We set the different ratios of hot data to cold data while leaving the other parameters fixed. The energy consumption of the three strategies is evaluated according to the different ratios. The common parameters used in the following three experiments are listed in Table 27.

In Table 27, the ratios of the other seasons with the different characteristics are the same as the Spring season, with the $s$ in the notation $\eta_{s}$ is respectively replaced by $\mathrm{m}, \mathrm{a}$, and $\mathrm{w}$.

Results obtained from simulation experiments when the ratio of hot data to cold data is set as 4:6 are shown in Table 28.

As shown in the Table 28, when the ratio of the hot data to the cold data is 4:6, the energy consumed by the K-ear is least, and the most is the Hadoop-default. While the energy consumed by the SEA is also fall in between. Compared to the Hadoop-default, the average energy consumption saved by the K-ear is about $42 \%$, while compared to the SEA, the saved energy consumption is also about $11 \%$. Moreover, we also have found that the system utilization has little impact on the energy consumption for the three algorithms.

Results obtained from simulation experiments when the ratio of hot data to cold data is set as 3:7 are shown in Table 29 .

As shown in the Table 29, when the ratio of the hot data to the cold data is 3:7, the energy consumed by the K-ear is least, and the most is the Hadoop-default. While the energy consumed by the SEA is also fall in between. Compared to the Hadoop-default, the average energy consumption saved by the K-ear is about $42 \%$, while compared to the SEA, the saved energy consumption is also about $2 \%$, which is almost the same. Moreover, we also have found that the system utilization has little impact on the energy consumption for the three algorithms.

Results obtained from simulation experiments when the ratio of hot data to cold data is set as 2:8 are shown in Table 30 .

As shown in the Table 30, when the ratio of the hot data to the cold data is 2:8, the energy consumed by the SEAis least, and the most is the Hadoop-default. While the energy consumed by the proposed K-ear is fall in between. Compared to the Hadoop-default, the average energy consumption saved by the K-ear is about $42 \%$, while compared to the SEA, the energy consumed by K-ear is increased is about 7\%. Moreover, we also have found that the system utilization has little impact on the energy consumption for the three algorithms in this experiment.

As shown in the above comparative experimental results, it can be seen that when the ratio of hot data to cold data is higher, the K-ear strategy performs better than the SEA algorithm. When the ratio is 4:6, K-ear has an obvious advantage over SEA. When the ratio is 3:7, the performance of K-ear and SEA are almost the same. When the ratio is 2:8, SEA slightly outperforms K-ear.

\section{Conclusions and Future Work}

An energy-aware data clustering strategy, K-ear, is proposed in this paper, in which the seasonal and tidal characteristics of data access are extracted thoroughly. The machine learning algorithm is applied to cluster data into different categories, and based on the categories, the data are stored in different storage zones. During the different time zones, some storage zones run in high performance mode to satisfy the performance requirement and the remaining storage zones run in low energy consumption mode to save energy consumption. To analyze and evaluate the energy efficiency of the proposed K-ear strategy, the famous classical SEA strategy and default data placement strategy in Hadoop are used for comparison, and mathematical models are constructed for the three strategies. Moreover, substantial simulation experiments are conducted in the CloudSimDisk simulator from different perspectives with different ratios. Compared with the mainstream data placement strategy (Hadoop default), K-ear and SEA are more energy efficient. The proposed K-ear strategy outperforms the classical SEA algorithm in most cases. Only when the ratio of hot data is very low is SEA more energy-efficient than K-ear. As a whole, compared with the other evaluated 
algorithms, the proposed K-ear data clustering storing strategies extracted the data access characteristics more thoroughly and classified the data into fine granularity categories, which will achieve more energy consumption savings with different disk running modes. Furthermore, as algorithm of SEA outperform the algorithms of Greedy [41], SP (Sort Partitions) [42], HP (Hybrid Partition), [42] and PVFS (Parallel Virtual File System)[43]. And the Hadoop default is more energy efficient than the algorithm of Datacenter without energy management. One part of our future work, we will explore how to combine the advantages of the different data placement strategies to achieve higher energy consumption reduction in cloud storage systems.

\section{CRediT author statement}

Xindong You: Conceptualization, Methodology, Validation, Writing original draft, Funding acquisition. Tian Sun: Formal analysis, Investigation, Data curation, Writing - review \& editing. Dawei Sun: Validation, Investigation, Writing - review \& editing. Xueqiang Lv: Conceptualization Supervision, Writing - review \& editing, Funding acquisition. Xunyun Liu: Data curation, Investigation, Writing - review \& editing. Rajkumar Buyya: Conceptualization, Writing - review \& editing, Supervision, Funding acquisition.

\section{Acknowledgments}

This work is supported by National Natural Science Foundation of China under Grants (No. 61671070, 61972364.), National Language Committee of China under Grants ZDI135-53, Australian Research Council (ARC) Discovery Project and Project of Developing University Intension for Improving the Level of Scientific Research-No.2019KYNH226, Qin Xin Talents Cultivation Program, Beijing Information Science \& Technology University No.QXTCP B201908.

\section{References}

[1] G.S. Aujla, N.Kumar. MEnSuS: An efficient scheme for energy management with sustainability of cloud data centers in edge-cloud environment. Future Generation Computer Systems. 86(9): 1279--1300. 2018.

[2] R. Kaushik. Energy management costs for a data center. USA Patent, Current Assignee: International Business Machines Corp. Granted in 2018.

[3] B. Varghese, R. Buyya. Next generation cloud computing: New trends and research directions. Future Generation Computer Systems. 79(3): 849-861. 2018.

[4] M. Mills, “The Cloud Begins with Coal: Big Data, Big Networks, Big Infrastructure, and Big Power,” in National Mining Association \& American Coalition for clean Coal Electricity, 2013.

[5] A.R.Dehghani-Sanij, E.Tharumalinga, M.B Dusseault, R.Fraser. Study of Energy Storage Systems and Environmental Challenges of Batteries. Renewable and Sustainable Energy Reviews. 104 (4) : 192-208, 2019.

[6] D.Popa, F.Pop, C. Serbanescu, A. Castiglione. Deep learning model for home automation and energy reduction in a smart home environment platform. Neural Computing and Applications. 31(5) : 1317-1337. 2019.

[7] Envantage. New Report Reveals Warehouses Overspend on Energy by £190m. Data by World Resources Institute. (14th August, 2013). Retrieved September 13.

[8] Uzaman, Sardar Khaliq, et al. A systems overview of commercial data centers: initial energy and cost analysis. International Journal of Information Technology and Web Engineering (IJITWE) 14.1 (2019): 42-65.

[9] Marahatta, Avinab, et al. Classification-based and energy-efficient dynamic task scheduling scheme for virtualized cloud data center. IEEE Transactions on Cloud Computing (2019).

[10] Lin, Hsueh-Yuan, and Sheng-Yuan Yang. A smart cloud-based energy data mining agent using big data analysis technology. Smart Science 7.3 (2019): 175-183. 
[11] Asgari, Sahar, et al. Hybrid surrogate model for online temperature and pressure predictions in data centers. Future Generation Computer Systems (2020).

[12] T. Xie, SEA: A Striping-Based Energy-Aware Strategy for Data Placement in RAID-Structured Storage Systems. IEEE Transactions on Computers, Vol.57, No.6, June 2008.

[13] R.T.Kaushik, and M. Bhandarkar. GreenHDFS: towards an energy-conserving, storage-efficient, hybrid Hadoop compute cluster, in Proc. of the 2010 international conference on Power aware computing and systems. HotPower '10, USENIX Association, Berkeley, CA,USA, 1-9, 2010.

[14] R. T.Kaushik., L.Cherkasova., R.Campbell, and R.Nahrstedt, Lightning: self-adaptive, energy-conserving, multi-zoned, commodity green cloud storage system, in Proc. of the 19th ACM International Symposium on High Performance Distributed Computing. 2010, HPDC '10.ACM, New York, NY, USA, 332-35.

[15] X.D. You, C. Dong, L. Zhou, J.Huang and C.F.Jiang. Anticipation-based Green Data Classification Strategy in Cloud Storage System. Appl.Math.Inf.SCi.9, No.4, 2151-2160 (2015).

[16] T. Zhang, B. Liao, H. Sun, F.G. Li, J.H. Ji. Energy-efficient algorithm based on data classification for cloud storage system. Journal of Computer Application, 2014, 34(8):2267-2273.

[17] X.L. Xu, G. Yang, L.J. Li, R.C. Wang. Dynamic data aggregation algorithm for data centers of green cloud computing. Systems Engineering and Electronics. Vol.34, No.9, September 2012.

[18] S.Q. Long. Research on Data Layout Strategies for Cloud Storage System. PHD thesis, South China University of Technology. 2014.

[19] B. Liao, J.Yu, H. Sun et al. Energy-efficient algorithms for distributed storage system based on data storage structure reconfiguration. Journal of Computer Research and Development, 2013, 50 (1) :3-18.

[20] R. Reddy, A. Kathpal, J. Basak, R. Katz. Data layout for power efficient archival storage systems. HotPower'15 Proceedings of the workshop on Power-Aware Computing and Systems. 2015.

[21] Z.Song. T.Wang,.T.Li, G. Yu. Energy Consumption Optimization Data Placement Algorithm for MapReduce System. Journal of Software, 2015, 26(8): 2091-2110.

[22] S.P. Ahuja, K. Muthiah. Survey of State-of-Art in Green Cloud Computing. International Journal of Green Computing (IJGC) 7(1). 2016.

[23] R. Yadav, Z, . "MeReg: Managing energy-SLA tradeoff for green mobile cloud computing." Wireless Communications and Mobile Computing 2017 (2017).

[24] R.Yadav, W.Zhang, H. Chen, T.Guo. (2017, August). Mums: Energy-aware vm selection scheme for cloud data center. In 2017 28th International Workshop on Database and Expert Systems Applications (DEXA) (pp. 132-136). IEEE.

[25] R.Yadav, et al. "Adaptive energy-aware algorithms for minimizing energy consumption and SLA violation in cloud computing." IEEE Access 6 (2018): 55923-55936.

[26] R.Yadav, et al. "An adaptive heuristic for managing energy consumption and overloaded hosts in a cloud data center." Wireless Networks 26.3 (2020): 1905-1919.

[27] Q.Wu, Q.Y. Deng, L. Ganesh. Dynamo: Facebook’s Data Center- Wide Power Management System.2016 ACM/IEEE 43rd Annual International Symposium on Computer Architecture (ISCA). 2016.

[28] R. Yadav, W.Z.Zhang, O. Kaiwartya, P.R.Singh, et al. Adaptive energy-aware algorithms for minimizing energy consumption and SLA violation in cloud computing, IEEE Access, 2018. Volume (6):55923-55936.

[29] L.Z.Wang, Y. Ma, J.N.Yan, V.Chang, A. Y. Zomaya. pipsCloud: High-performance cloud computing for remote sensing 
big data management and processing, Future Generation Computer Systems, Volume 78, Part 1, 2018: 353-368.

[30] J. Song, H.Y.He, G. Yu, J.M. Pierson. Modulo Based Data Placement Algorithm for Energy Consumption Optimization of MapReduce System. Journal of Grid Computing .2018, 16(3):409-424.

[31] X.T.Tran, T.V.Do, C. Rotter, D. Hwang. A New Data Layout Scheme for Energy-Efficient MapReduce Processing Tasks. Journal of Grid Computing. 2018, 16(2): 285-298.

[32] Y.Ebadi, N.J. Navimipour. An energy-aware method for data replication in the cloud environments using a Tabu search and particle swarm optimization algorithm. Wiley Online Library, 28 August, 2018.

[33] R. Vales, J. Moura, R. Marinheiro. Energy-aware and adaptive fog storage mechanism with data replication ruled by spatio-temporal content popularity. Journal of Network and Computer Applications. 2019, 135(6): 84-96.

[34] C.L.Li, Y.P. Wang, Y.Chen, Y.L. Luo. Energy-efficient Fault-tolerant Replica Management Policy with Deadline and Budget Constraints in Edge-cloud Environment. Journal of Network and Computer Applications. 2019,143(1): 152-166.

[35] A.A. Khan, M.Zakarya, R.Khan, I. Rahman, M.Khan. An Energy, Performance Efficient Resource Consolidation Scheme for Heterogeneous Cloud Datacenters. Volume 150, 15 January 2020, 102497

[36] B. Louis, K. Mitra, S. Saguna, and C. Åhlund, CloudSimDisk: Energy-Aware Storage Simulation in CloudSim, Proceedings of the 2015 IEEE/ACM 8th International Conference on Utility and Cloud Computing (UCC), Limassol, Cyprus.

[37] R. Calheiros, R. Ranjan, A. Beloglazov, C. Rose, and R. Buyya, CloudSim: A Toolkit for Modeling and Simulation of Cloud Computing Environments and Evaluation of Resource Provisioning Algorithms, Software: Practice and Experience, Volume 41, Number 1, Pages: 23-50, ISSN: 0038-0644, Wiley Press, New York, USA, January 2011.

[38] S. Chatradhi. Hard Drive for Low Power Energy Efficiency in Disk Storage, (c) 2009 Hitachi Global Storage Technologies https://e3s-center.berkeley.edu/wp-content/uploads/2017/07/Sridhar\%20Energy efficient hdd June2009.pdf

[39] A. Hylick, R. Sohan, A. Rice and B. Jones, "An Analysis of Hard Drive Energy Consumption," 2008 IEEE International Symposium on Modeling, Analysis and Simulation of Computers and Telecommunication Systems, Baltimore, MD, 2008, pp. 1-10, doi: 10.1109/MASCOT.2008.4770567.

[40] J.Zedlewski, S. Sobti, N. Garg, Z. Fengzhou,et al.Modeling Hard-Disk Power Consumption Proceedings of FAST ’03:2nd USENIX Conference onFile and Storage TechnologiesSan Francisco, CA, USAMarch 31-April 2, 2003.

[41] R.L. Graham, Bounds on Multiprocessing Timing Anomalies, SIAM J. Applied Math., vol. 7, no. 2, pp. 416-429, 1969.

[42] L.W. Lee, P. Scheuermann, and R. Vingralek, File Assignment in Parallel I/O Systems with Minimal Variance of Service Time, IEEE Trans. Computers, vol. 49, no. 2, pp. 127-140, Feb. 2000.

[43] R. Latham, N. miller, R. Ross, and P. Carns, A Next-Generation Parallel File System for Linux Clusters: An Introduction to the Second Parallel Virtual File System, Linux World Magazine, pp. 56-59, Jan. 2004.

This article is protected by copyright. All rights reserved. 
Table 1 Summary of the reviewed related work compared with our work

\begin{tabular}{|c|c|c|c|c|c|}
\hline \multirow[t]{2}{*}{ Work } & \multirow[t]{2}{*}{ Name } & \multirow{2}{*}{$\begin{array}{l}\text { Access } \\
\text { frequency }\end{array}$} & \multicolumn{2}{|c|}{ Periodic access characteristics } & \multirow[t]{2}{*}{ Zones divided } \\
\hline & & & $\begin{array}{c}\text { Seasonal } \\
\text { characteristics }\end{array}$ & $\begin{array}{c}\text { Tidal } \\
\text { characteristics }\end{array}$ & \\
\hline T.Xie[12] & SEA & $\checkmark$ & - & - & Hot Disk Zone, Cold Disk Zone \\
\hline $\begin{array}{c}\text { R.T. Kaushiketal } \\
\text { [13] }\end{array}$ & $\begin{array}{c}\text { GreenHD } \\
\text { FS }\end{array}$ & $\checkmark$ & - & - & Hot Zone, Cold Zone \\
\hline $\begin{array}{c}\text { R.T. Kaushiketal } \\
\text { [14] }\end{array}$ & Lightning & $\checkmark$ & - & - & Hot Zone, Cold Zone \\
\hline $\begin{array}{c}\text { X,D.You et al } \\
\text { [15] }\end{array}$ & AGDC & $\checkmark$ & $\checkmark$ & - & ColdZone, Seasonal Hot Zone, HotZone \\
\hline Z. Tao etal. [16] & - & $\checkmark$ & - & - & HotZone, ColdZone and Reduplication Zone \\
\hline X.L.Xu [17] & - & $\checkmark$ & - & - & Dynamically Aggregating \\
\hline S.Q.Long [18] & SFLS & $\checkmark$ & - & - & HotFiles Zone, BigFiles Zone \\
\hline Our Work & K-ear & $\checkmark$ & $\checkmark$ & $\checkmark$ & $\begin{array}{c}\text { Five Big Zones (according to seasonal characteristics) with } \\
\text { Fifteen Small Zones (according to tidal characteristics } \\
\text { furthermore) }\end{array}$ \\
\hline
\end{tabular}

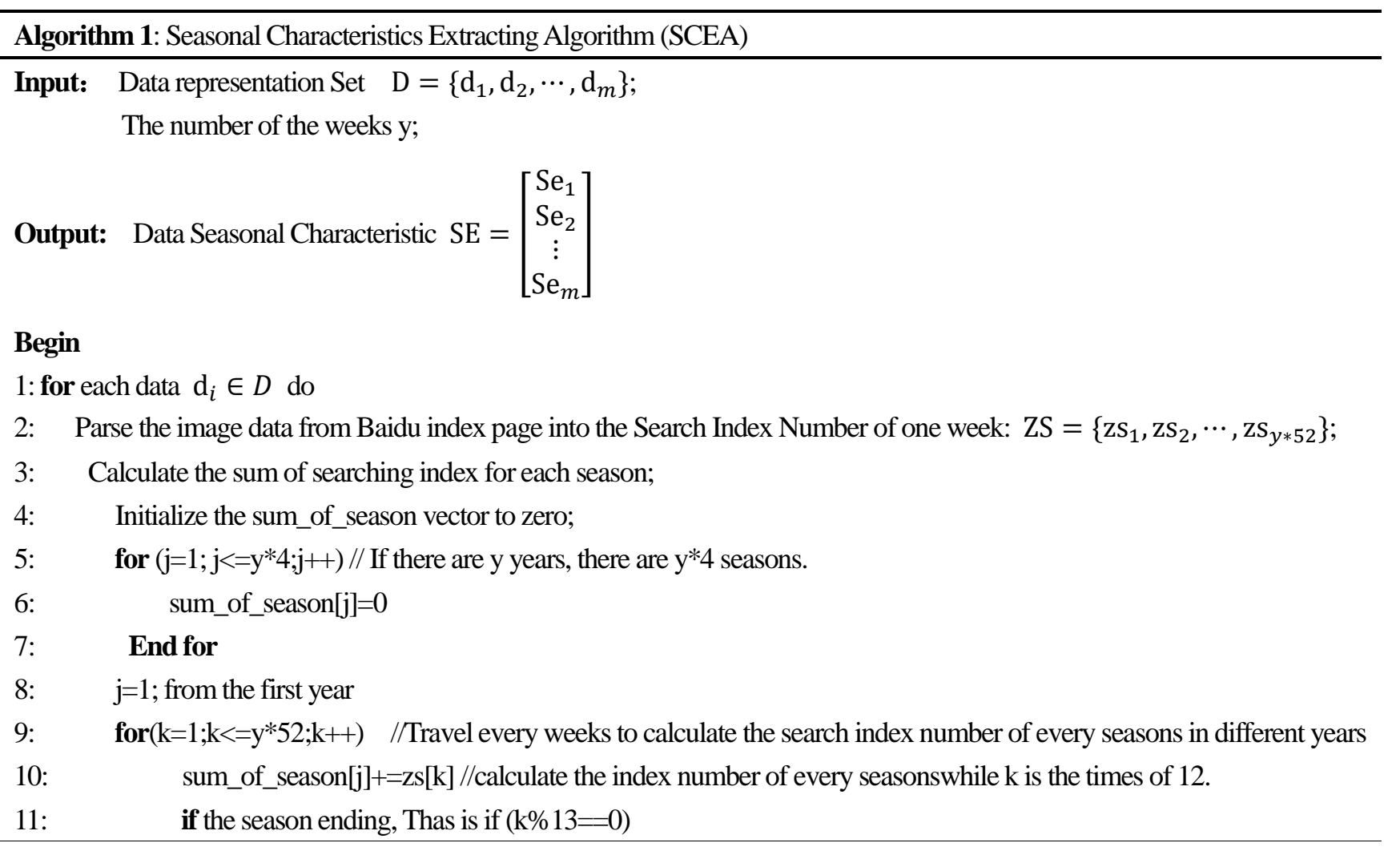



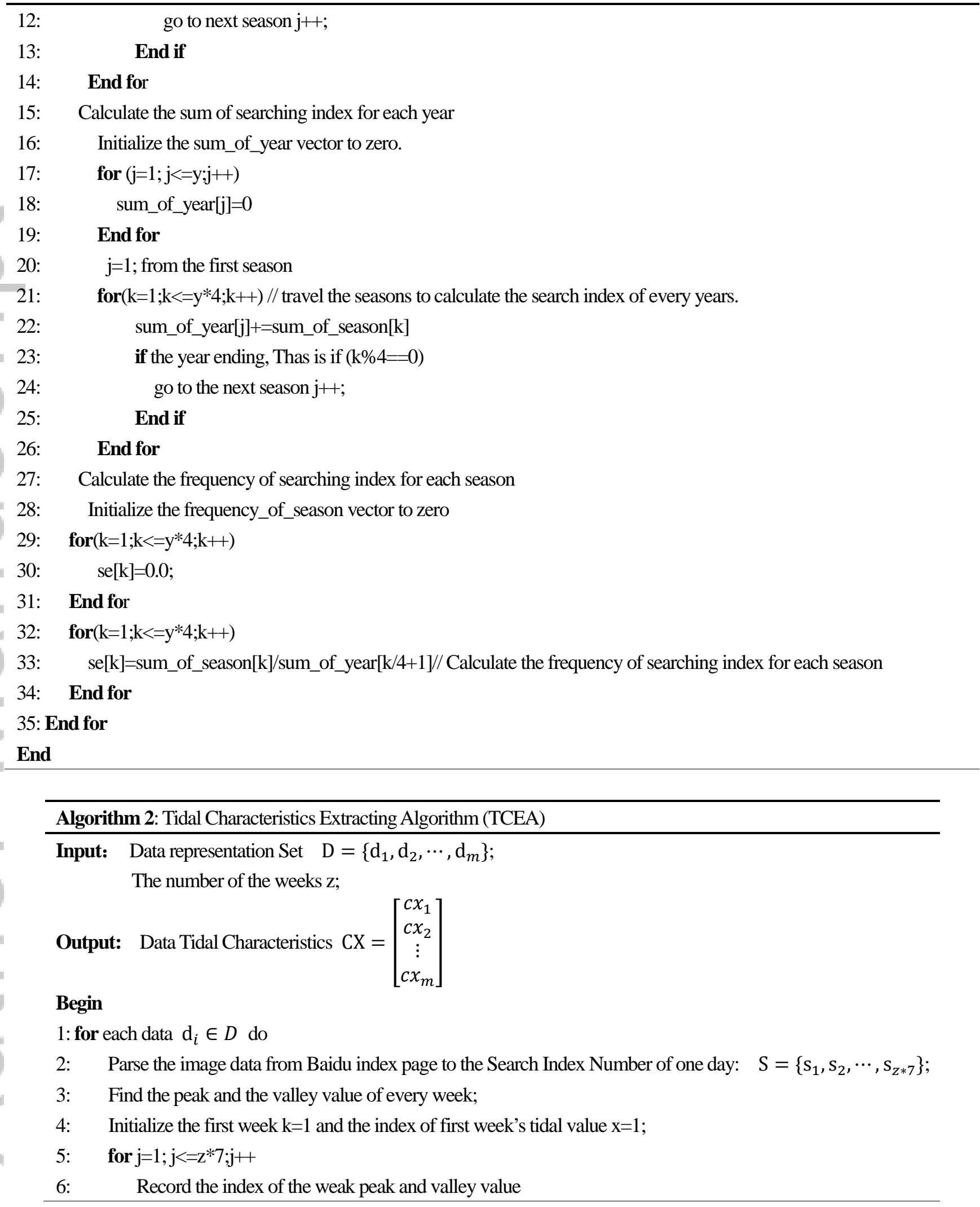


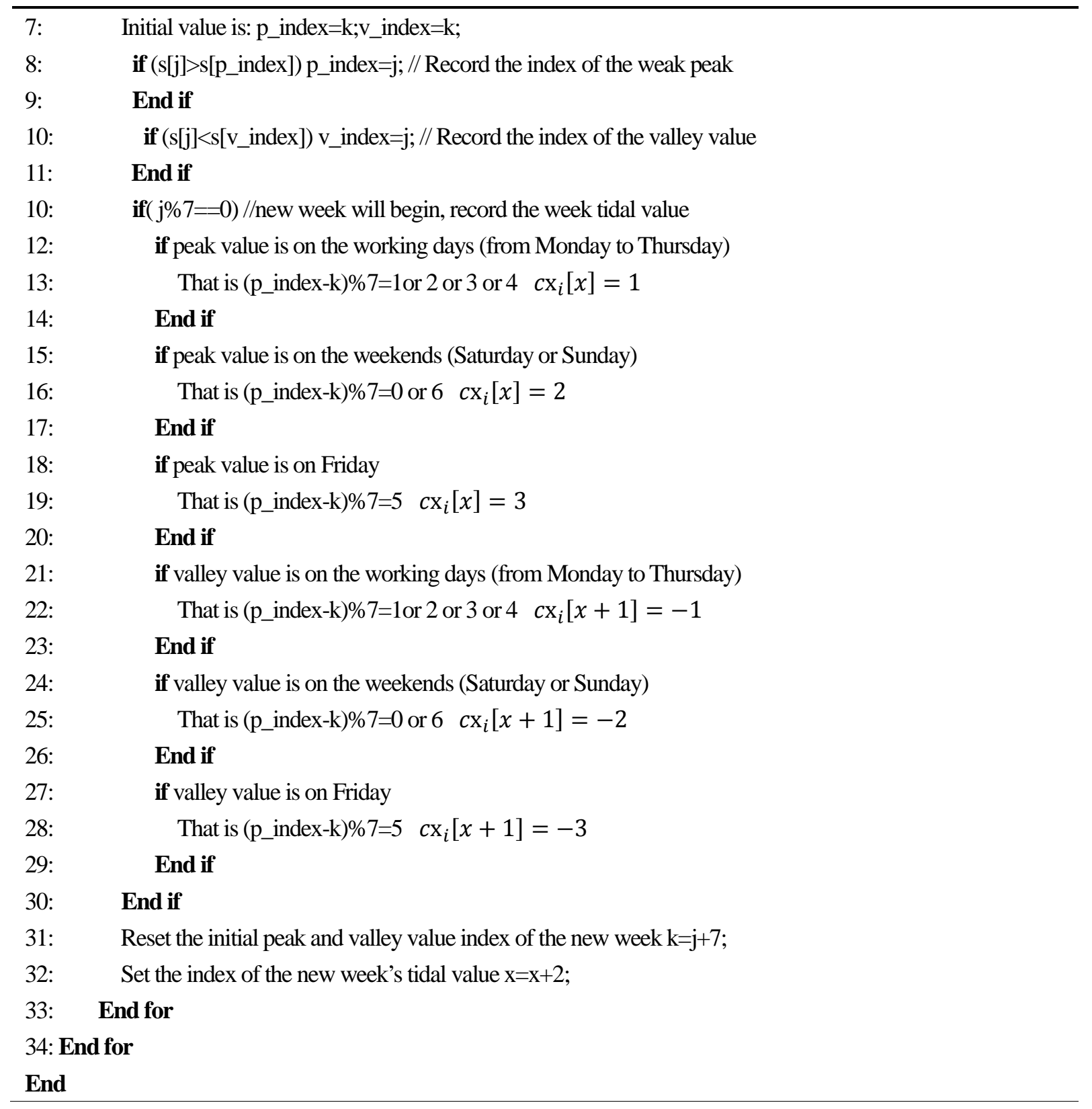

Table 2: Symbols in Algorithms related to the seasonal data proportion

\begin{tabular}{|c|c|c|c|}
\hline Symbol & Meaning & Symbol & Meaning \\
\hline \multirow[t]{3}{*}{$\eta_{s}$} & \multirow{3}{*}{$\begin{array}{l}\text { Ratio of data with spring } \\
\text { characteristics }\end{array}$} & $\eta_{s-\mathrm{w}}$ & Ratio of data with Spring and work characteristics \\
\hline & & $\eta_{s-\mathrm{h}}$ & Ratio of data with Spring and entertainment characteristics \\
\hline & & $\eta_{s-0}$ & Ratio of data with Spring but without characteristics \\
\hline \multirow[t]{3}{*}{$\eta_{o}$} & \multirow{3}{*}{$\begin{array}{l}\text { Ratio of data without } \\
\text { seasonal characteristics }\end{array}$} & $\eta_{o-\mathrm{w}}$ & Ratio of data with work but without seasonal characteristics \\
\hline & & $\eta_{o-h}$ & Ratio of data with entertainment but without seasonal characteristics \\
\hline & & $\eta_{o-0}$ & Ratio of data without seasonal and tidal characteristics \\
\hline
\end{tabular}


Table 3: The number of disks to store the data with tidal characteristics of every seasonal zone

\begin{tabular}{cl}
\hline Parameters & \multicolumn{1}{c}{ Meanings } \\
\hline$\eta_{s-\mathrm{w}} \times n_{1}$ & The number of disks to store the data with spring and work characteristics \\
\hline$\eta_{s-\mathrm{h}} \times n_{1}$ & The number of disks to store the data with spring and entertainment characteristics \\
\hline$\eta_{s-\mathrm{o}} \times n_{1}$ & The number of disks to store the data with spring but without tidal characteristics \\
\hline$\eta_{o-\mathrm{w}} \times n_{5}$ & The number of disks to store the data with work but without seasonal characteristics \\
\hline$\eta_{o-\mathrm{h}} \times n_{5}$ & $\begin{array}{l}\text { The number of disks to store the data with entertainment but without seasonal } \\
\text { characteristics }\end{array}$ \\
\hline$\eta_{o-\mathrm{o}} \times n_{5}$ & The number of disks to store the data without work seasonal characteristics
\end{tabular}

Algorithm 3: Energy-aware data classification based on K-means (K-ear)

Input: Data related characteristics: a collection of $m$ data in the set D, and the corresponding parameters: $\eta_{s}, \eta_{m}, \eta_{a}, \eta_{w}, \eta_{o}$ and $\eta_{s-\mathrm{w}}, \eta_{s-\mathrm{h}}, \eta_{s-\mathrm{o}}, \eta_{m-\mathrm{w}}, \eta_{m-\mathrm{h}}, \eta_{m-\mathrm{o}}, \eta_{a-\mathrm{w}}, \eta_{a-\mathrm{h}}, \eta_{\mathrm{a}-\mathrm{o}}, \eta_{w-\mathrm{w}}, \eta_{w-\mathrm{h}}, \eta_{w-o}, \eta_{o-\mathrm{w}}, \eta_{o-\mathrm{h}}, \eta_{o-0}$.

The exacted data characteristics: the output of the algorithm1 and algorithm 3: Data Tidal Characteristics $\mathrm{CX}=\left[\begin{array}{c}c x_{1} \\ c x_{2} \\ \vdots \\ c x_{m}\end{array}\right]$, and the

Data Seasonal Characteristics $\mathrm{SE}=\left[\begin{array}{c}\mathrm{Se}_{1} \\ \mathrm{Se}_{2} \\ \vdots \\ \mathrm{Se}_{m}\end{array}\right]$

Disk related characteristics: A disk array DISK with $n$ size, every disk with 2-speed mode.

Output: Allocation of the data on the corresponding storage zones.

Begin

1: for each data $\mathrm{d}_{i} \in D$ do

2: Based on the Data Seasonal Characteristics $\mathrm{SE}=\left[\begin{array}{c}\mathrm{Se}_{1} \\ \mathrm{Se}_{2} \\ \vdots \\ \mathrm{Se}_{m}\end{array}\right]$, and use the K-means clustering algorithm to classify the data into five classes firstly;

3: if $\mathrm{d}_{i}$ has the spring characteristics (Class 1)

4: $\quad$ place it evenly into the following storage zone $S=\left\{s_{1}, s_{2}, \cdots, s_{n 1}\right\}$

(Storage Zone 1)

5: $\quad$ based on the Data Tidal Characteristics $C X_{1}=\left[\begin{array}{c}c x_{11} \\ c x_{12} \\ \vdots \\ c x_{1 \eta_{s} \times m}\end{array}\right]$, and use the K-means clustering algorithm to classify the data 
into three classes;

6: $\quad$ If $\mathrm{d}_{i}$ has the working day characteristics (Sub_Class 1-1)

7: place the data into the $1 \sim \eta_{s-\mathrm{w}} \times n_{1}$ disks evenly(Storage Zone 1-1) // disks numbered by ordering

8:

\section{End if}

9: $\quad$ If $\mathrm{d}_{i}$ has the holiday characteristics(Sub_Class 1-2)

10: $\quad$ place the data into the $\eta_{s-\mathrm{w}} \times n_{1}+1 \sim \eta_{s-\mathrm{w}} \times n_{1}+\eta_{s-\mathrm{h}} \times n_{1}$ disks evenly; (Storage Zone 1-2) // disks numbered by ordering

11: $\quad$ End if

12: $\quad$ If $\mathrm{d}_{i}$ has no working and holiday characteristics (Sub_Class 1-3)

13: $\quad$ place the data into the $\eta_{s-\mathrm{w}} \times n_{1}+\eta_{s-\mathrm{h}} \times n_{1}+1 \sim n_{1}$ disks evenly (Storage Zone 1-3) // disks numbered by ordering

\section{4: $\quad$ End if}

\section{5: End if}

16: if $\mathrm{d}_{i}$ has the summer characteristics (Class 2)

17: place it evenly into the following storage zone $M=\left\{\mathrm{m}_{1}, \mathrm{~m}_{2}, \cdots \mathrm{m}_{n 2}\right\}$;

(Storage Zone 2)

18: based on the Data Tidal Characteristics $C X_{2}=\left[\begin{array}{c}c x_{21} \\ c x_{22} \\ \vdots \\ c x_{2 \eta_{m} \times m}\end{array}\right]$, and use the K-means clustering algorithm to classify the data into three classes;

19:

If $\mathrm{d}_{i}$ has the working day characteristics (Sub_Class 2-1) place the data into the $1 \sim \eta_{m-\mathrm{w}} \times n_{2}$ disks evenly; // disks numbered by ordering

(Storage Zone 2-1)

21:

End if place the data into the $\eta_{m-\mathrm{w}} \times n_{2}+1 \sim \eta_{m-\mathrm{w}} \times n_{2}+\eta_{m-\mathrm{h}} \times n_{2}$ disks evenly; (Storage Zone 2-2) $/ /$ disks numbered by ordering

\section{End if}

25:

If $\mathrm{d}_{i}$ has no working and holiday characteristics (Sub_Class 2-3) place the data into the $\eta_{m-\mathrm{w}} \times n_{2}+\eta_{m-\mathrm{h}} \times n_{2}+1 \sim n_{2}$ disks evenly; // disks numbered by ordering (Storage Zone 2-3)

\section{End if}

\section{8: $\quad$ End if}

29: if $\mathrm{d}_{i}$ has the autumn characteristics (Class 3)

30: place it evenly into the following storage zone $A=\left\{\mathrm{a}_{1}, \mathrm{a}_{2}, \cdots \mathrm{a}_{n 3}\right\}$;

(Storage Zone 3) 
31: based on the Data Tidal Characteristics $\mathrm{CX}_{3}=\left[\begin{array}{c}c x_{31} \\ c x_{32} \\ \vdots \\ c x_{3 \eta_{a} \times m}\end{array}\right]$, and use the K-means clustering algorithm to classify the data into three classes;

If $\mathrm{d}_{i}$ has the working day characteristics (Sub_Class 3-1) place the data into the $1 \sim \eta_{a-\mathrm{w}} \times n_{3}$ disks evenly; // disks numbered by ordering

(Storage Zone 3-1)

\section{End if} // disks numbered by ordering

\section{End if}

38:

If $\mathrm{d}_{i}$ has no working and holiday characteristics (Sub_Class 3-3) place the data into the $\eta_{a-\mathrm{w}} \times n_{3}+\eta_{s-\mathrm{h}} \times n_{3}+1 \sim n_{3}$ disks evenly // disks numbered by ordering

\section{(Storage Zone 3-3)}

\section{0: $\quad$ End if}

41: $\quad$ End if

42: $\quad$ if $d_{i}$ has winter characteristics; (Class 4)

43: $\quad$ place it evenly into the following storage zone $W=\left\{w_{1}, w_{2}, \cdots, w_{n 4}\right\}$

(Storage Zone 4)

44: $\quad$ based on the Data Tidal Characteristics $\mathrm{CX}_{4}=\left[\begin{array}{c}c x_{41} \\ c x_{42} \\ \vdots \\ c x_{4 \eta_{w} \times m}\end{array}\right]$, and use the K-means clustering algorithm to classify the data into three classes;

45: $\quad$ If $\mathrm{d}_{i}$ has the working day characteristics (Sub_Class 4-1)

46: place the data into the $1 \sim \eta_{w-\mathrm{w}} \times n_{4}$ disks evenly // disks numbered by ordering;

\section{(Storage Zone 4-1)}

47:

\section{End if}

If $\mathrm{d}_{i}$ has the holiday characteristics (Sub_Class 4-2)

place the data into the $\eta_{w-w} \times n_{4}+1 \sim \eta_{w-w} \times n_{4}+\eta_{w-\mathrm{h}} \times n_{4}$ disks evenly; (Storage Zone 4-2) // disks numbered by ordering

50:

\section{End if}

51: $\quad$ If $\mathrm{d}_{i}$ has no working and holiday characteristics (Sub_Class 4-3) place the data into the $\eta_{w-\mathrm{w}} \times n_{4}+\eta_{w-\mathrm{h}} \times n_{4}+1 \sim n_{4}$ disks evenly // disks numbered by ordering (Storage Zone 4-3) 
55: $\quad$ if $\mathrm{d}_{i}$ has no seasonal characteristics (Class 5)

place it evenly into the following storage zone $O=\left\{\mathrm{o}_{1}, \mathrm{o}_{2}, \cdots \mathrm{o}_{n 5}\right\}$;

(Storage Zone 5)

56: based on the Data Tidal Characteristics $\mathrm{CX}_{5}=\left[\begin{array}{c}c x_{51} \\ c x_{52} \\ \vdots \\ c x_{5 \eta_{o} \times m}\end{array}\right]$, and use the K-means clustering algorithm to classify the data into three classes;

If $\mathrm{d}_{i}$ has the working day characteristics (Sub_Class 5-1)

58: $\quad$ place the data into the $1 \sim \eta_{o-\mathrm{w}} \times n_{5}$ disks evenly; // disks numbered by ordering

(Storage Zone 5-1)

59: $\quad$ End if

60: $\quad$ If $\mathrm{d}_{i}$ has the holiday characteristics (Sub_Class 5-2)

61: $\quad$ place the data into the $\eta_{o-\mathrm{w}} \times n_{5}+1 \sim \eta_{o-\mathrm{w}} \times n_{5}+\eta_{o-\mathrm{h}} \times n_{5}$ disks evenly; (Storage Zone 5-2) // disks numbered by ordering

62:

\section{End if}

If $\mathrm{d}_{i}$ has no working and holiday characteristics (Sub_Class 5-3) place the data into the $\eta_{o-\mathrm{w}} \times n_{5}+\eta_{o-\mathrm{h}} \times n_{5}+1 \sim n_{5}$ disks evenly; // disks numbered by ordering (Storage Zone 5-3)

65:

\section{End if}

66: End if

67: End for

End

Table 4 The number of disks running in different modes during different time zones

\begin{tabular}{|c|c|c|}
\hline Time Zone & The number of disks running in high mode & The number of disks running in low mode \\
\hline Workdays in Spring & $\begin{aligned} \mathrm{H} 1=\eta_{s-\mathrm{w}} \times n_{1} & +\eta_{s-\mathrm{o}} \times n_{1}+\eta_{s-\mathrm{w}} \\
& \times n_{5}+\eta_{s-\mathrm{o}} \times n_{5}\end{aligned}$ & $\mathrm{~L} 1=\eta_{s-\mathrm{h}} \times n_{1}+n_{2}+n_{3}+n_{4}+\eta_{s-\mathrm{h}} \times n_{5}$ \\
\hline Weekends in Spring & $\begin{aligned} \mathrm{H} 2=\eta_{s-h} \times n_{1} & +\eta_{s-\mathrm{o}} \times n_{1}+\eta_{s-\mathrm{h}} \times n_{5} \\
& +\eta_{s-\mathrm{o}} \times n_{5}\end{aligned}$ & $\mathrm{~L} 2=\eta_{s-\mathrm{w}} \times n_{1}+n_{2}+n_{3}+n_{4}+\eta_{s-\mathrm{w}} \times n_{5}$ \\
\hline Workdays in Summer & $\begin{aligned} \mathrm{H} 3=\eta_{s-\mathrm{w}} \times n_{2} & +\eta_{s-\mathrm{o}} \times n_{2}+\eta_{s-\mathrm{w}} \\
& \times n_{5}+\eta_{s-\mathrm{o}} \times n_{5}\end{aligned}$ & $\mathrm{~L} 3=\eta_{s-\mathrm{h}} \times n_{2}+n_{1}+n_{3}+n_{4}+\eta_{s-\mathrm{h}} \times n_{5}$ \\
\hline Weekends in Summer & $\begin{aligned} \mathrm{H} 4=\eta_{s-h} \times n_{2} & +\eta_{s-\mathrm{o}} \times n_{2}+\eta_{s-\mathrm{h}} \times n_{5} \\
& +\eta_{s-\mathrm{o}} \times n_{5}\end{aligned}$ & $\mathrm{~L} 3=\eta_{s-\mathrm{w}} \times n_{2}+n_{1}+n_{3}+n_{4}+\eta_{s-w} \times n_{5}$ \\
\hline Workdays in Autumn & $\begin{aligned} \mathrm{H} 5=\eta_{s-\mathrm{w}} \times n_{3} & +\eta_{s-\mathrm{o}} \times n_{3}+\eta_{s-\mathrm{w}} \\
& \times n_{5}+\eta_{s-\mathrm{o}} \times n_{5}\end{aligned}$ & $\mathrm{~L} 5=\eta_{s-\mathrm{h}} \times n_{3}+n_{1}+n_{2}+n_{4}+\eta_{s-\mathrm{h}} \times n_{5}$ \\
\hline
\end{tabular}




\begin{tabular}{lrl}
\hline Weekends in Autumn & H6 $=\eta_{s-\mathrm{h}} \times n_{3}+\eta_{s-\mathrm{o}} \times n_{3}+\eta_{s-\mathrm{h}} \times n_{5}$ & $\mathrm{~L} 6=\eta_{s-\mathrm{w}} \times n_{3}+n_{1}+n_{2}+n_{4}+\eta_{s-\mathrm{w}} \times n_{5}$ \\
& $+\eta_{s-\mathrm{o}} \times n_{5}$
\end{tabular}

Table 5: Meaning of the parameters of the whole system in mathematic models

\begin{tabular}{|c|c|}
\hline Paramter & Meaning \\
\hline$\tau^{h}$ & Transfer rate of disks running in high mode (MB/second) \\
\hline $\mathrm{p}^{h}$ & Energy consuming rate of disks running in high mode during active status $(\mathrm{J} / \mathrm{second})$ \\
\hline $\mathrm{i}^{h}$ & Energy consuming rate of disks running in high mode during idle status (J/second) \\
\hline$\tau^{l}$ & Transfer rate of disks running in low mode (MB/second) \\
\hline $\mathrm{p}^{l}$ & Energy consuming rate of disks running in low mode during active status (J/second) \\
\hline $\mathrm{i}^{l}$ & Energy consuming rate of disks running in low mode during idle status (J/second) \\
\hline $\mathrm{s}^{\prime}$ & The average size of the dataset $(\mathrm{MB})$ \\
\hline $\mathrm{e}_{\text {total }}$ & The total enery consumption $(\mathrm{J})$ \\
\hline$e_{h}$ & Energy consumed by all of the disks running in high mode (J) \\
\hline$e_{l}$ & Energy consumed by all of the disks running in low mode (J) \\
\hline$e_{h}^{\text {active }}$ & Energy consumed by all of the disks running in high mode during active status (J) \\
\hline$e_{h}^{i d l e}$ & Energy consumed by all of the disks running in high mode during idle status (J) \\
\hline$e_{l}^{\text {active }}$ & Energy consumed by all of the disks running in low mode during active status (J) \\
\hline$e_{l}^{i d l e}$ & Energy consumed by all of the disks running in low mode during idle status (J) \\
\hline$t_{h}^{\text {active }}$ & Total time of disks running in high mode with active status (Second) \\
\hline$t_{h}^{i d l e}$ & Total time of disks running in high mode with idle status (Second) \\
\hline$t_{l}^{\text {active }}$ & Total time of disks running in low mode with active status (Second) \\
\hline$t_{l}^{\text {idle }}$ & Total time of disks running in low mode with idle status (Second) \\
\hline$T$ & al service time of a disk (Second /disk) \\
\hline
\end{tabular}

Table 6: Meaning of the parameters about the spring season in mathematic models

\begin{tabular}{|c|c|}
\hline spring_work_t $t_{h}^{\text {active }}$ & $\begin{array}{l}\text { Total time of disks running in high mode with active status during workdays in Spring } \\
\text { (Second) }\end{array}$ \\
\hline spring_holiday_t $t_{h}^{\text {active }}$ & $\begin{array}{l}\text { Total time of disks running in high mode with active status during weekends in Spring } \\
\text { (Second) }\end{array}$ \\
\hline spring_work_t thle & Total time of disks running in high mode with idle status during workdays in Spring (Second) \\
\hline spring_holiday_t $t_{h}^{i d l e}$ & Total time of disks running in high mode with idle status during weekends in Spring (Second) \\
\hline spring_work_t $t_{l}^{\text {active }}$ & Total time of disks running in low mode with active status during workdays in Spring \\
\hline
\end{tabular}




\begin{tabular}{|c|c|}
\hline & (Second) \\
\hline spring_holiday_t $t_{l}^{\text {active }}$ & $\begin{array}{l}\text { Total time of disks running in low mode with active status during weekends in Spring } \\
\text { (Second) }\end{array}$ \\
\hline spring_work_t $t_{l}^{\text {idle }}$ & Total time of disks running in low mode with idle status during workdays in Spring (Second) \\
\hline spring_holiday_t $t_{l}^{\text {idle }}$ & Total time of disks running in low mode with idle status during weekends in Spring (Second) \\
\hline spring_work_e $\mathrm{e}_{\text {total }}$ & Energy consumption during workdays in Spring $(\mathrm{J})$ \\
\hline spring_holiday_e $\mathrm{e}_{\text {total }}$ & Energy consumption during weekends in Spring $(\mathrm{J})$ \\
\hline spring_work_e $e_{h}^{\text {active }}$ & $\begin{array}{l}\text { Energy Consumption of disks running in high mode with active status during workdays in } \\
\text { Spring (J) }\end{array}$ \\
\hline spring_holiday_e $e_{h}^{\text {active }}$ & $\begin{array}{l}\text { Energy Consumption of disks running in high mode with active status during weekends in } \\
\text { Spring (J) }\end{array}$ \\
\hline spring_work_e $e_{l}^{\text {active }}$ & $\begin{array}{l}\text { Energy Consumption of disks running in low mode with active status during workdays in } \\
\text { Spring }(J)\end{array}$ \\
\hline spring_holiday_e $e_{l}^{\text {active }}$ & $\begin{array}{l}\text { Energy Consumption of disks running in low mode with active status during weekends in } \\
\text { Spring }(J)\end{array}$ \\
\hline spring_work_ $e_{h}^{\text {idle }}$ & $\begin{array}{l}\text { Energy Consumption of disks running in high mode with idle status during workdays in } \\
\text { Spring (J) }\end{array}$ \\
\hline spring_holiday_ $e_{h}^{i d l e}$ & $\begin{array}{l}\text { Energy Consumption of disks running in high mode with idle status during weekends in } \\
\text { Spring }(\mathrm{J})\end{array}$ \\
\hline spring_work_ $e_{l}^{\text {idle }}$ & $\begin{array}{l}\text { Energy Consumption of disks running in low mode with idle status during workdays in } \\
\text { Summer (J) }\end{array}$ \\
\hline spring_holiday_e $e_{l}^{i d l e}$ & $\begin{array}{l}\text { Energy Consumption of disks running in low mode with idle status during weekends in } \\
\text { Summer (J) }\end{array}$ \\
\hline spring_work_T & Total service time of a disk during workdays in Spring (Second /disk) = 5T/28 \\
\hline spring_holiday_T & Total service time of a disk during weekends in Spring (Second /disk) = 2T/28 \\
\hline$S_{-} W_{h}^{n}$ & Total access times in high mode during workdays in Spring \\
\hline$S_{-} W_{l}^{n}$ & Total access times in low mode during workdays in Spring \\
\hline$S_{-} H_{h}^{n}$ & Total access times in high mode during weekends in Spring \\
\hline$S_{-} H_{l}^{n}$ & Total access times in low mode during weekends in Spring \\
\hline
\end{tabular}

Table7 Software and Hardware Configuration in the Experiments

\begin{tabular}{lc}
\hline Equipment/Software & type/version \\
\hline CPU & Intel(R) Core(TM) i5-4590 CPU @ 3.30GHz 3.3 GHz \\
\hline Memory Size & $4.0 \mathrm{~GB}$ \\
\hline Hard Disk & 1TB(TOSHIBA DT01ACA100 ATA Device) \\
\hline
\end{tabular}




\begin{tabular}{|c|c|c|c|}
\hline \multicolumn{2}{|l|}{ Network Card } & & k PCIe GBE Family Controller \\
\hline \multicolumn{2}{|c|}{ Operating System } & & Windows 10 \\
\hline \multicolumn{2}{|c|}{ Energy-aware Disk Simulator } & & CloudSimDisk1.0 \\
\hline \multicolumn{2}{|c|}{ Cloud Environment Simulator } & & CloudSim 4.0 \\
\hline \multicolumn{2}{|c|}{ Programming Platform } & & Eclipse-Java-Luna-SR2 \\
\hline \multicolumn{4}{|c|}{ Table 8: General parameters value in the simulation experiments } \\
\hline Paramter & Value & Paramter & Value \\
\hline $\mathrm{p}^{h}$ & $30.26 \mathrm{~J} /$ Second & $i^{l}$ & $2.17 \mathrm{~J} /$ Second \\
\hline$i^{h}$ & $5.26 \mathrm{~J} /$ Second & $\tau^{l}$ & 9.3Mb/Second \\
\hline$\tau^{\mathrm{h}}$ & $31 \mathrm{Mb} /$ Second & $\mathrm{n}$ & 1000 \\
\hline $\mathrm{p}^{l}$ & $21.33 \mathrm{~J} /$ Second & $\mathrm{T}$ & 31536000 Second \\
\hline
\end{tabular}

Table 9 Parameters and values set in this experiment

\begin{tabular}{|c|c|c|c|}
\hline Parameter & value & Parameter & value \\
\hline \multirow{3}{*}{$\begin{array}{l}\text { Ratio of the data with } \\
\text { Spring } \\
\text { characteristics } \eta_{s}\end{array}$} & \multirow[t]{3}{*}{0.2} & Ratio of data with Spring and workday characteristics $\eta_{s-w}$ & 0.3 \\
\hline & & Ratio of data with Spring and weekend characteristics $\eta_{s-\mathrm{h}}$ & 0.3 \\
\hline & & $\begin{array}{l}\text { Ratio of data with Spring characteristics but without tidal } \\
\text { characteristics } \eta_{s-o}\end{array}$ & 0.4 \\
\hline \multirow{3}{*}{$\begin{array}{l}\text { Ratio of the data } \\
\text { without seasonal } \\
\text { characteristics } \eta_{o}\end{array}$} & \multirow[t]{3}{*}{0.2} & $\begin{array}{l}\text { Ratio of data with workday characteristics but without seasonal } \\
\text { characteristics } \eta_{o-w}\end{array}$ & 0.3 \\
\hline & & $\begin{array}{l}\text { Ratio of data with weekend characteristics but without seasonal } \\
\text { characteristics } \eta_{o-h}\end{array}$ & 0.3 \\
\hline & & Ratio of data without seasonal and tidal characteristics $\eta_{o-o}$ & 0.4 \\
\hline
\end{tabular}

Table 10: Energy consumption of the three data placement strategies when the ratio of the high disk utilization to the whole system utilization is 1.6

\begin{tabular}{llll}
\hline $\begin{array}{l}\text { System } \\
\text { utilization }\end{array}$ & $\begin{array}{l}\text { Energy consumption of Kear } \\
(\mathrm{KJ})\end{array}$ & $\begin{array}{l}\text { Energy consumption of Hadoop-default } \\
(\mathrm{KJ})[13]\end{array}$ & $\begin{array}{l}\text { Energy consumption of SEA(KJ) } \\
{[12]}\end{array}$ \\
\hline 0.1 & 95998.62547 & 165958.2 & 107658.606 \\
\hline 0.11 & 96026.6813 & 165966.084 & 107683.3049 \\
\hline 0.12 & 96054.73713 & 165973.968 & 107708.0039 \\
\hline 0.13 & 96082.79295 & 165981.852 & 107732.7029 \\
\hline 0.14 & 96110.84878 & 165989.736 & 107757.4019 \\
\hline 0.15 & 96138.90461 & 165997.62 & 107782.1009 \\
\hline 0.16 & 96166.96043 & 166005.504 & 107806.7999 \\
\hline
\end{tabular}




\begin{tabular}{llll}
\hline 0.17 & 96195.01626 & 166013.388 & 107831.4989 \\
\hline 0.18 & 96223.07209 & 166021.272 & 107856.1979 \\
\hline 0.19 & 96251.12792 & 166029.156 & 107880.8969 \\
\hline 0.2 & 96279.18374 & 166037.04 & 107905.5959 \\
\hline 0.21 & 96307.23957 & 166044.924 & 107930.2949 \\
\hline 0.22 & 96335.2954 & 166052.808 & 107954.9939 \\
\hline 0.23 & 96363.35123 & 166060.692 & 107979.6929 \\
\hline 0.24 & 96391.40705 & 166068.576 & 108004.3919 \\
\hline 0.25 & 96419.46288 & 166076.46 & 108029.0909 \\
\hline 0.26 & 96447.51871 & 166084.344 & 108053.7899 \\
\hline 0.27 & 96475.57453 & 166092.228 & 108078.4889 \\
\hline 0.28 & 96503.63036 & 166100.112 & 108103.1879 \\
\hline 0.29 & 96531.68619 & 166107.996 & 108127.8869 \\
\hline 0.3 & 96559.74202 & 166115.88 & 108152.5859 \\
\hline 0.31 & 96587.79784 & 166123.764 & 108177.2849 \\
\hline 0.32 & 96615.85367 & 166131.648 & 108201.9838 \\
\hline 0.33 & 96643.9095 & 166139.532 & 108226.6828 \\
\hline 0.34 & 96671.96532 & 166147.416 & 108251.3818 \\
\hline 0.35 & 96700.02115 & 166155.3 & 108276.0808 \\
\hline 0.36 & 96728.07698 & 166163.184 & 108300.7798 \\
\hline 0.37 & 96756.13281 & 166171.068 & 108325.4788 \\
\hline 0.38 & 96784.18863 & 166178.952 & 108350.1778 \\
\hline 0.39 & 96812.24446 & 166186.836 & 108374.8768 \\
\hline & & &
\end{tabular}

Table 11: Energy consumption of the three data placement strategies when the ratio of the high disk utilization to the whole system utilization is 1.8

\begin{tabular}{llll}
\hline $\begin{array}{l}\text { System } \\
\text { utilization }\end{array}$ & $\begin{array}{l}\text { Energy consumption of Kear } \\
(\mathrm{KJ})\end{array}$ & $\begin{array}{l}\text { Energy consumption of Hadoop-default } \\
(\mathrm{KJ})[13]\end{array}$ & $\begin{array}{l}\text { Energy consumption of SEA(KJ) } \\
{[12]}\end{array}$ \\
\hline 0.1 & 95998.72826 & 165958.2 & 107647.5193 \\
\hline 0.11 & 96026.79436 & 165966.084 & 107671.1096 \\
\hline 0.12 & 96054.86047 & 165973.968 & 107694.7 \\
\hline 0.13 & 96082.92657 & 165981.852 & 107718.2903 \\
\hline 0.14 & 96110.99268 & 165989.736 & 107741.8806 \\
\hline 0.15 & 96139.05878 & 165997.62 & 107765.4709 \\
\hline 0.16 & 96167.12489 & 166005.504 & 107789.0613 \\
\hline 0.17 & 96195.19099 & 166013.388 & 107812.6516 \\
\hline 0.18 & 96223.2571 & 166021.272 & 107836.2419 \\
\hline 0.19 & 96251.32321 & 166029.156 & 107859.8323 \\
\hline
\end{tabular}

This article is protected by copyright. All rights reserved. 


\begin{tabular}{llll}
\hline 0.2 & 96279.38931 & 166037.04 & 107883.4226 \\
\hline 0.21 & 96307.45542 & 166044.924 & 107907.0129 \\
\hline 0.22 & 96335.52152 & 166052.808 & 107930.6033 \\
\hline 0.23 & 96363.58763 & 166060.692 & 107954.1936 \\
\hline 0.24 & 96391.65373 & 166068.576 & 107977.7839 \\
\hline 0.25 & 96419.71984 & 166076.46 & 108001.3742 \\
\hline 0.26 & 96447.78595 & 166084.344 & 108024.9646 \\
\hline 0.27 & 96475.85205 & 166092.228 & 108048.5549 \\
\hline 0.28 & 96503.91816 & 166100.112 & 108072.1452 \\
\hline 0.3 & 96531.98426 & 166107.996 & 108095.7356 \\
\hline 0.31 & 96560.05037 & 166115.88 & 108119.3259 \\
\hline 0.32 & 96588.11647 & 166123.764 & 108142.9162 \\
\hline 0.33 & 96616.18258 & 166131.648 & 108166.5065 \\
\hline 0.34 & 96644.24868 & 166139.532 & 108190.0969 \\
\hline 0.35 & 96672.31479 & 166147.416 & 108213.6872 \\
\hline 0.36 & 96700.3809 & 166155.3 & 108237.2775 \\
\hline 0.37 & 96728.447 & 166163.184 & 108260.8679 \\
\hline 0.38 & 96756.51311 & 166171.068 & 108284.4582 \\
\hline 0.39 & 96784.57921 & 166178.952 & 108308.0485 \\
\hline & 96812.64532 & 166186.836 & 108331.6389 \\
\hline
\end{tabular}

Table 12: Energy consumption of the three data placement strategies when the ratio of the high disk utilization to the whole system utilization is 2.0

\begin{tabular}{llll}
\hline $\begin{array}{l}\text { System } \\
\text { utilization }\end{array}$ & $\begin{array}{l}\text { Energy consumption of } \\
\text { K-ear }(\mathrm{KJ})\end{array}$ & $\begin{array}{l}\text { Energy consumption of Hadoop-default } \\
(\mathrm{KJ})[13]\end{array}$ & $\begin{array}{l}\text { Energy consumption of } \\
\text { SEA(KJ) [12] }\end{array}$ \\
\hline 0.1 & 95998.83104 & 165958.2 & 107636.4326 \\
\hline 0.11 & 96026.90742 & 165966.084 & 107658.9143 \\
\hline 0.12 & 96054.98381 & 165973.968 & 107681.396 \\
\hline 0.13 & 96083.06019 & 165981.852 & 107703.8776 \\
\hline 0.14 & 96111.13658 & 165989.736 & 107726.3593 \\
\hline 0.15 & 96139.21296 & 165997.62 & 107748.841 \\
\hline 0.16 & 96167.28934 & 166005.504 & 107771.3226 \\
\hline 0.17 & 96195.36573 & 166013.388 & 107793.8043 \\
\hline 0.18 & 96223.44211 & 166021.272 & 107816.286 \\
\hline 0.19 & 96251.5185 & 166029.156 & 107838.7676 \\
\hline
\end{tabular}

This article is protected by copyright. All rights reserved. 


\begin{tabular}{llll}
\hline 0.2 & 96279.59488 & 166037.04 & 107861.2493 \\
\hline 0.21 & 96307.67126 & 166044.924 & 107883.7309 \\
\hline 0.22 & 96335.74765 & 166052.808 & 107906.2126 \\
\hline 0.23 & 96363.82403 & 166060.692 & 107928.6943 \\
\hline 0.24 & 96391.90042 & 166068.576 & 107951.1759 \\
\hline 0.25 & 96419.9768 & 166076.46 & 107973.6576 \\
\hline 0.26 & 96448.05318 & 166084.344 & 107996.1393 \\
\hline 0.27 & 96476.12957 & 166092.228 & 108018.6209 \\
\hline 0.28 & 96504.20595 & 166100.112 & 108041.1026 \\
\hline 0.29 & 96532.28234 & 166107.996 & 108063.5843 \\
\hline 0.3 & 96560.35872 & 166115.88 & 108086.0659 \\
\hline 0.31 & 96588.4351 & 166123.764 & 108108.5476 \\
\hline 0.32 & 96616.51149 & 166131.648 & 108131.0292 \\
\hline 0.33 & 96644.58787 & 166139.532 & 108153.5109 \\
\hline 0.34 & 96672.66426 & 166147.416 & 108175.9926 \\
\hline 0.35 & 96700.74064 & 166155.3 & 108198.4742 \\
\hline 0.36 & 96728.81702 & 166163.184 & 108220.9559 \\
\hline 0.37 & 96756.89341 & 166171.068 & 108243.4376 \\
\hline 0.38 & 96784.96979 & 166178.952 & 108265.9192 \\
\hline 0.39 & 96813.04618 & 166186.836 & 108288.4009 \\
\hline & & &
\end{tabular}

Table 13: General parameters' values for the different ratios of the data with seasonal characteristics and the data without seasonal characteristics

\begin{tabular}{ll}
\hline \multicolumn{1}{c}{ Parameter } & Value \\
\hline Ratio of the data with Spring and workday characteristics $\eta_{s-\mathrm{w}}$ & 0.3 \\
\hline Ratio of the data with Spring and weekend characteristics $\eta_{s-\mathrm{h}}$ & 0.3 \\
\hline Ratio of the data with Spring but without tidal characteristics $\eta_{s-\mathrm{o}}$ & 0.4 \\
\hline Ratio of the data with workday characteristics but without seasonal characteristics $\eta_{o-\mathrm{w}}$ & 0.3 \\
\hline
\end{tabular}

This article is protected by copyright. All rights reserved. 


\begin{tabular}{lc}
\hline Ratio of the data with weekend characteristics but without seasonal characteristics $\eta_{o-h}$ & 0.3 \\
\hline Ratio of the data without seasonal and tidal characteristics $\eta_{o-o}$ & 0.4 \\
\hline
\end{tabular}

Table 14: Seasonal characteristics related parameters’ value set in experiment 1

\begin{tabular}{cc} 
parameter & value \\
\hline Ratio of the data with Spring characteristics $\eta_{\mathrm{s}}$ & 0.15 \\
\hline Ratio of the data with Summer characteristics $\eta_{\mathrm{m}}$ & 0.15 \\
\hline Ratio of the data with Autumn characteristics $\eta_{\mathrm{a}}$ & 0.15 \\
\hline Ratio of the data with Winter characteristics $\eta_{\mathrm{w}}$ & 0.15 \\
\hline Ratio of the data without seasonal characteristics $\eta_{\mathrm{o}}$ & 0.4 \\
\hline
\end{tabular}

Table 15: Energy consumption of the three data placement strategies in experiment 1

\begin{tabular}{llll}
\hline $\begin{array}{l}\text { System } \\
\text { utilization }\end{array}$ & $\begin{array}{l}\text { Energy consumption of Kear } \\
(\mathrm{KJ})\end{array}$ & $\begin{array}{l}\text { Energy consumption of Hadoop-default } \\
(\mathrm{KJ})[13]\end{array}$ & $\begin{array}{l}\text { Energy consumption of SEA(KJ) } \\
{[12]}\end{array}$ \\
\hline 0.1 & 106182.9264 & 165958.2 & 107636.4326 \\
\hline 0.11 & 106206.2268 & 165966.084 & 107658.9143 \\
\hline 0.12 & 106229.5272 & 165973.968 & 107681.396 \\
\hline 0.13 & 106252.8276 & 165981.852 & 107703.8776 \\
\hline 0.14 & 106276.128 & 165989.736 & 107726.3593 \\
\hline 0.15 & 106299.4285 & 165997.62 & 107748.841 \\
\hline 0.16 & 106322.7289 & 166005.504 & 107771.3226 \\
\hline 0.17 & 106346.0293 & 166013.388 & 107793.8043 \\
\hline 0.18 & 106369.3297 & 166021.272 & 107816.286 \\
\hline 0.19 & 106392.6301 & 166029.156 & 107838.7676 \\
\hline 0.2 & 106415.9305 & 166037.04 & 107861.2493 \\
\hline 0.21 & 106439.2309 & 166044.924 & 107883.7309 \\
\hline 0.22 & 106462.5313 & 166052.808 & 107906.2126 \\
\hline 0.23 & 106485.8317 & 166060.692 & 107928.6943 \\
\hline 0.24 & 106509.1321 & 166068.576 & 107951.1759 \\
\hline 0.25 & 106532.4325 & 166076.46 & 107973.6576 \\
\hline 0.26 & 106555.7329 & 166084.344 & 107996.1393 \\
\hline 0.27 & 106579.0333 & 166092.228 & 108018.6209 \\
\hline 0.28 & 106602.3337 & 166100.112 & 108041.1026 \\
\hline 0.29 & 106625.6341 & 166107.996 & 108063.5843 \\
\hline 0.3 & 106648.9345 & 166115.88 & 108086.0659 \\
\hline 0.31 & 106672.2349 & 166123.764 & 108108.5476 \\
\hline & &
\end{tabular}

This article is protected by copyright. All rights reserved. 


\begin{tabular}{llll}
\hline 0.32 & 106695.5353 & 166131.648 & 108131.0292 \\
\hline 0.33 & 106718.8357 & 166139.532 & 108153.5109 \\
\hline 0.34 & 106742.1361 & 166147.416 & 108175.9926 \\
\hline 0.35 & 106765.4365 & 166155.3 & 108198.4742 \\
\hline 0.36 & 106788.7369 & 166163.184 & 108220.9559 \\
\hline 0.37 & 106812.0373 & 166171.068 & 108243.4376 \\
\hline 0.38 & 106835.3377 & 166178.952 & 108265.9192 \\
\hline 0.39 & 106858.6381 & 166186.836 & 108288.4009 \\
\hline
\end{tabular}

Table 16: Seasonal characteristics related parameters' value set in experiment 2

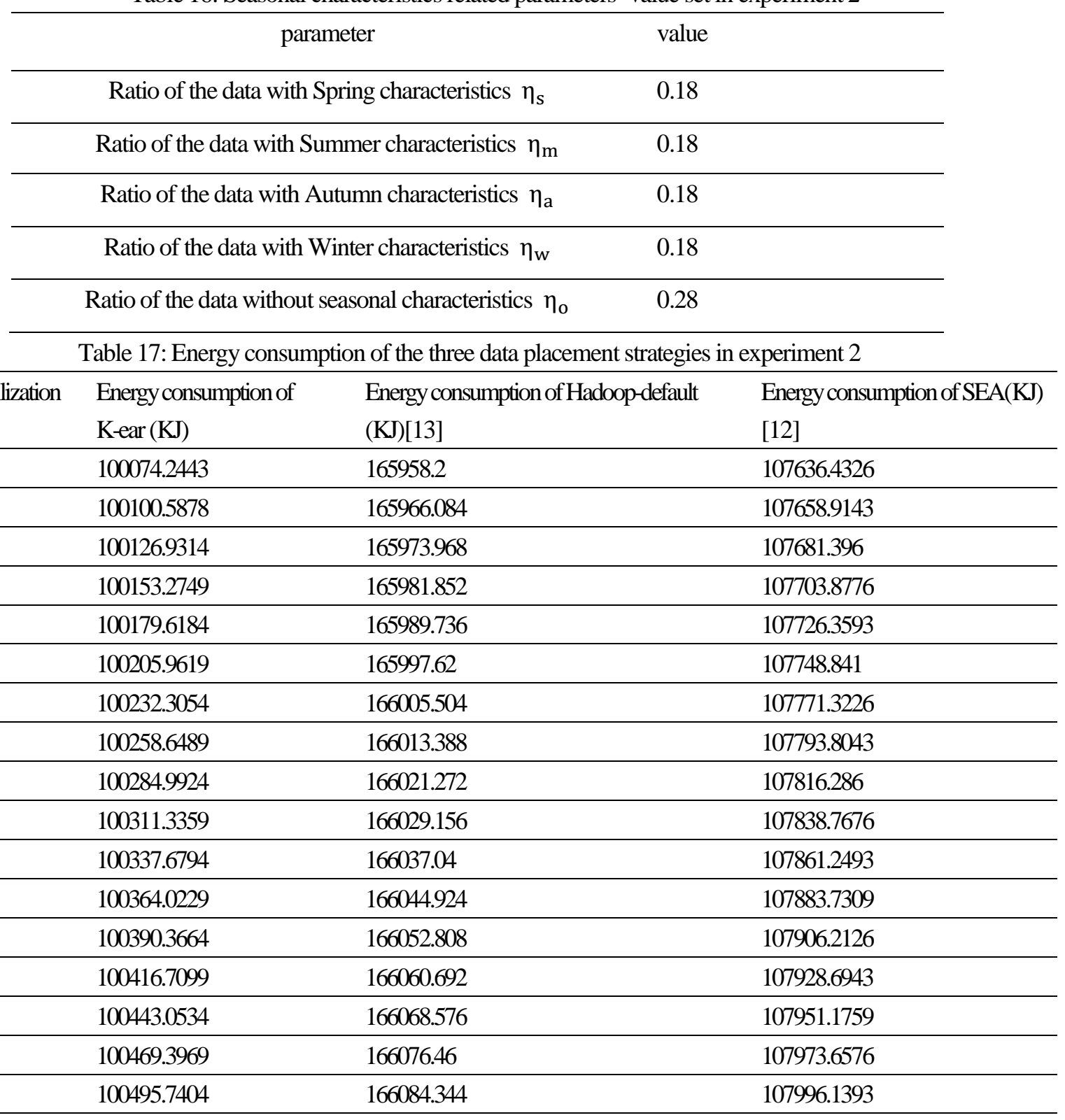




\begin{tabular}{llll}
\hline 0.27 & 100522.0839 & 166092.228 & 108018.6209 \\
\hline 0.28 & 100548.4275 & 166100.112 & 108041.1026 \\
\hline 0.29 & 100574.771 & 166107.996 & 108063.5843 \\
\hline 0.3 & 100601.1145 & 166115.88 & 108086.0659 \\
\hline 0.31 & 100627.458 & 166123.764 & 108108.5476 \\
\hline 0.32 & 100653.8015 & 166131.648 & 108131.0292 \\
\hline 0.33 & 100680.145 & 166139.532 & 108153.5109 \\
\hline 0.34 & 100706.4885 & 166147.416 & 108175.9926 \\
\hline 0.35 & 100732.832 & 166155.3 & 108198.4742 \\
\hline 0.36 & 100759.1755 & 166163.184 & 108220.9559 \\
\hline 0.37 & 100785.519 & 166171.068 & 108243.4376 \\
\hline 0.38 & 100811.8625 & 166178.952 & 108265.9192 \\
\hline 0.39 & 100838.206 & 166186.836 & 108288.4009 \\
\hline
\end{tabular}

Table 18: Seasonal characteristics related parameters' value set in experiment 3

\begin{tabular}{cc}
\hline parameter & value \\
\hline Ratio of the data with Spring characteristics $\eta_{\mathrm{s}}$ & 0.12 \\
\hline Ratio of the data with Summer characteristics $\eta_{\mathrm{m}}$ & 0.12 \\
\hline Ratio of the data with Autumn characteristics $\eta_{\mathrm{a}}$ & 0.12 \\
\hline Ratio of the data with Winter characteristics $\eta_{\mathrm{w}}$ & 0.12 \\
\hline Ratio of the data without seasonal characteristics $\eta_{\mathrm{o}}$ & 0.52 \\
\hline
\end{tabular}

Table 19: Energy consumption of the three data placement strategies in experiment 3.

\begin{tabular}{llll}
\hline Systemutilization & $\begin{array}{l}\text { Energy consumption of Kear } \\
(\mathrm{KJ})\end{array}$ & $\begin{array}{l}\text { Energy consumption of } \\
\text { Hadoop-default }(\mathrm{KJ})[13]\end{array}$ & $\begin{array}{l}\text { Energy consumption of } \\
\text { SEA(KJ) [12] }\end{array}$ \\
\hline 0.1 & 112284.6623 & 165958.2 & 107636.4326 \\
\hline 0.11 & 112304.225 & 165966.084 & 107658.9143 \\
\hline 0.12 & 112323.7877 & 165973.968 & 107681.396 \\
\hline 0.13 & 112343.3504 & 165981.852 & 107703.8776 \\
\hline 0.14 & 112362.913 & 165989.736 & 107726.3593 \\
\hline 0.15 & 112382.4757 & 165997.62 & 107748.841 \\
\hline 0.16 & 112402.0384 & 166005.504 & 107771.3226 \\
\hline 0.17 & 112421.6011 & 166013.388 & 107793.8043 \\
\hline 0.18 & 112441.1638 & 166021.272 & 107816.286 \\
\hline 0.19 & 112460.7264 & 166029.156 & 107838.7676 \\
\hline 0.2 & 112480.2891 & 166037.04 & 107861.2493 \\
\hline
\end{tabular}

This article is protected by copyright. All rights reserved. 


\begin{tabular}{llll}
\hline 0.21 & 112499.8518 & 166044.924 & 107883.7309 \\
\hline 0.22 & 112519.4145 & 166052.808 & 107906.2126 \\
\hline 0.23 & 112538.9772 & 166060.692 & 107928.6943 \\
\hline 0.24 & 112558.5398 & 166068.576 & 107951.1759 \\
\hline 0.25 & 112578.1025 & 166076.46 & 107973.6576 \\
\hline 0.26 & 112597.6652 & 166084.344 & 107996.1393 \\
\hline 0.27 & 112617.2279 & 166092.228 & 108018.6209 \\
\hline 0.28 & 112636.7906 & 166100.112 & 108041.1026 \\
\hline 0.29 & 112656.3532 & 166107.996 & 108063.5843 \\
\hline 0.3 & 112675.9159 & 166115.88 & 108086.0659 \\
\hline 0.31 & 112695.4786 & 166123.764 & 108108.5476 \\
\hline 0.32 & 112715.0413 & 166131.648 & 108131.0292 \\
\hline 0.33 & 112734.6039 & 166139.532 & 108153.5109 \\
\hline 0.34 & 112754.1666 & 166147.416 & 108175.9926 \\
\hline 0.35 & 112773.7293 & 166155.3 & 108198.4742 \\
\hline 0.36 & 112793.292 & 166163.184 & 108220.9559 \\
\hline 0.37 & 112812.8547 & 166171.068 & 108243.4376 \\
\hline 0.38 & 112832.4173 & 166178.952 & 108265.9192 \\
\hline 0.39 & 112851.98 & 166186.836 & 108288.4009 \\
\hline & & &
\end{tabular}

Table 20: Common parameters used in the following three experiments

\begin{tabular}{cl}
\hline parameter & value \\
\hline Ratio of the data with Spring characteristics $\eta_{\mathrm{s}}$ & 0.2 \\
\hline Ratio of the data with Summer characteristics $\eta_{\mathrm{m}}$ & 0.2 \\
\hline Ratio of the data with Autumn characteristics $\eta_{\mathrm{a}}$ & 0.2 \\
\hline Ratio of the data with Winter characteristics $\eta_{\mathrm{w}}$ & 0.2 \\
\hline Ratio of the data without seasonal characteristics $\eta_{\mathrm{o}}$ & 0.2 \\
\hline Ratio of the high speed disk utilization to the system utilization & 2.0 \\
\hline Ratio of the hot data to the cold data & $4: 6$ \\
\hline
\end{tabular}

Table 21: Tidal characteristics related parameters used in the first experiment

\begin{tabular}{ll}
\hline \multicolumn{1}{c}{ Parameter } & value \\
\hline Ratio of the data with Spring and workday characteristics $\eta_{s-\mathrm{w}}$ & 0.2 \\
\hline Ratio of the data with Spring and weekend characteristics $\eta_{s-\mathrm{h}}$ & 0.2 \\
\hline
\end{tabular}

This article is protected by copyright. All rights reserved. 


\begin{tabular}{ll}
\hline Ratio of the data with Spring but without tidal characteristics $\eta_{s-\mathrm{o}}$ & 0.6 \\
\hline Ratio of the data with workday but without seasonal characteristics $\eta_{o-\mathrm{w}}$ & 0.2 \\
\hline Ratio of the data with weekend but without seasonal characteristics $\eta_{o-\mathrm{h}}$ & 0.2 \\
\hline Ratio of the data without seasonal and tidal characteristics $\eta_{o-\mathrm{o}}$ & 0.6 \\
\hline
\end{tabular}

Table 22: Energy consumption of the three data placement strategies when the ratio of the data with tidal characteristics to the data without tidal characteristics is 4:6

\begin{tabular}{|c|c|c|c|}
\hline System utilization & $\begin{array}{l}\text { Energy consumption of } \\
\text { Kear }(\mathrm{KJ})\end{array}$ & $\begin{array}{l}\text { Energy consumption of } \\
\text { Hadoop-default (KJ)[13] }\end{array}$ & $\begin{array}{l}\text { Energy consumption of } \\
\text { SEA(KJ) [12] }\end{array}$ \\
\hline 0.1 & 99880.22558 & 165958.2 & 107636.4326 \\
\hline 0.11 & 99906.65646 & 165966.084 & 107658.9143 \\
\hline 0.12 & 99933.08734 & 165973.968 & 107681.396 \\
\hline 0.13 & 99959.51822 & 165981.852 & 107703.8776 \\
\hline 0.14 & 99985.94909 & 165989.736 & 107726.3593 \\
\hline 0.15 & 100012.38 & 165997.62 & 107748.841 \\
\hline 0.16 & 100038.8108 & 166005.504 & 107771.3226 \\
\hline 0.17 & 100065.2417 & 166013.388 & 107793.8043 \\
\hline 0.18 & 100091.6726 & 166021.272 & 107816.286 \\
\hline 0.19 & 100118.1035 & 166029.156 & 107838.7676 \\
\hline 0.2 & 100144.5344 & 166037.04 & 107861.2493 \\
\hline 0.21 & 100170.9652 & 166044.924 & 107883.7309 \\
\hline 0.22 & 100197.3961 & 166052.808 & 107906.2126 \\
\hline 0.23 & 100223.827 & 166060.692 & 107928.6943 \\
\hline 0.24 & 100250.2579 & 166068.576 & 107951.1759 \\
\hline 0.25 & 100276.6888 & 166076.46 & 107973.6576 \\
\hline 0.26 & 100303.1196 & 166084.344 & 107996.1393 \\
\hline 0.27 & 100329.5505 & 166092.228 & 108018.6209 \\
\hline 0.28 & 100355.9814 & 166100.112 & 108041.1026 \\
\hline 0.29 & 100382.4123 & 166107.996 & 108063.5843 \\
\hline 0.3 & 100408.8431 & 166115.88 & 108086.0659 \\
\hline 0.31 & 100435.274 & 166123.764 & 108108.5476 \\
\hline 0.32 & 100461.7049 & 166131.648 & 108131.0292 \\
\hline 0.33 & 100488.1358 & 166139.532 & 108153.5109 \\
\hline 0.34 & 100514.5667 & 166147.416 & 108175.9926 \\
\hline 0.35 & 100540.9975 & 166155.3 & 108198.4742 \\
\hline 0.36 & 100567.4284 & 166163.184 & 108220.9559 \\
\hline 0.37 & 100593.8593 & 166171.068 & 108243.4376 \\
\hline
\end{tabular}

This article is protected by copyright. All rights reserved. 


\begin{tabular}{llll}
\hline 0.38 & 100620.2902 & 166178.952 & 108265.9192 \\
\hline 0.39 & 100646.721 & 166186.836 & 108288.4009 \\
\hline
\end{tabular}

Table 23: Tidal characteristics related parameters used in the second experiment

\begin{tabular}{ll}
\hline \multicolumn{1}{c}{ Parameter } & Value \\
\hline Ratio of the data with Spring and workday characteristics $\eta_{s-\mathrm{w}}$ & 0.25 \\
\hline Ratio of the data with Spring and weekend characteristics $\eta_{s-\mathrm{h}}$ & 0.25 \\
\hline Ratio of the data with Spring but without tidal characteristics $\eta_{s-\mathrm{o}}$ & 0.5 \\
\hline Ratio of the data with workday but without seasonal characteristics $\eta_{o-\mathrm{w}}$ & 0.25 \\
\hline Ratio of the data with weekend but without seasonal characteristics $\eta_{o-\mathrm{h}}$ & 0.25 \\
\hline Ratio of the data without seasonal and tidal characteristics $\eta_{o-\mathrm{o}}$ & 0.5 \\
\hline
\end{tabular}

Table 24: Energy consumption of the three data placement strategies when the ratio of the data with tidal characteristics to the data without tidal characteristics is 5:5

\begin{tabular}{llll}
\hline System utilization & $\begin{array}{l}\text { Energy consumption of Kear } \\
(\mathrm{KJ})\end{array}$ & $\begin{array}{l}\text { Energy consumption of Hadoop-default } \\
(\mathrm{KJ})[13]\end{array}$ & $\begin{array}{l}\text { Energy consumption of SEA(KJ) } \\
{[12]}\end{array}$ \\
\hline 0.1 & 97939.76338 & 165958.2 & 107636.4326 \\
\hline 0.11 & 97967.04052 & 165966.084 & 107658.9143 \\
\hline 0.12 & 97994.31766 & 165973.968 & 107681.396 \\
\hline 0.13 & 98021.5948 & 165981.852 & 107703.8776 \\
\hline 0.14 & 98048.87194 & 165989.736 & 107726.3593 \\
\hline 0.15 & 98076.14907 & 165997.62 & 107748.841 \\
\hline 0.16 & 98103.42621 & 166005.504 & 107771.3226 \\
\hline 0.17 & 98130.70335 & 166013.388 & 107793.8043 \\
\hline 0.18 & 98157.98049 & 166021.272 & 107816.286 \\
\hline 0.19 & 98185.25763 & 166029.156 & 107838.7676 \\
\hline 0.2 & 98212.53477 & 166037.04 & 107861.2493 \\
\hline 0.21 & 98239.8119 & 166044.924 & 107883.7309 \\
\hline 0.22 & 98267.08904 & 166052.808 & 107906.2126 \\
\hline 0.23 & 98294.36618 & 166060.692 & 107928.6943 \\
\hline 0.24 & 98321.64332 & 166068.576 & 107951.1759 \\
\hline 0.25 & 98348.92046 & 166076.46 & 107973.6576 \\
\hline 0.26 & 98376.19759 & 166084.344 & 107996.1393 \\
\hline 0.27 & 98403.47473 & 166092.228 & 108018.6209 \\
\hline 0.28 & 98430.75187 & 166100.112 & 108041.1026 \\
\hline 0.29 & 98458.02901 & 166107.996 & 108063.5843 \\
\hline & & & \\
\hline
\end{tabular}




\begin{tabular}{llll}
\hline 0.3 & 98485.30615 & 166115.88 & 108086.0659 \\
\hline 0.31 & 98512.58329 & 166123.764 & 108108.5476 \\
\hline 0.32 & 98539.86042 & 166131.648 & 108131.0292 \\
\hline 0.33 & 98567.13756 & 166139.532 & 108153.5109 \\
\hline 0.34 & 98594.4147 & 166147.416 & 108175.9926 \\
\hline 0.35 & 98621.69184 & 166155.3 & 108198.4742 \\
\hline 0.36 & 98648.96898 & 166163.184 & 108220.9559 \\
\hline 0.37 & 98676.24612 & 166171.068 & 108243.4376 \\
\hline 0.38 & 98703.52325 & 166178.952 & 108265.9192 \\
\hline
\end{tabular}

Table 25: Tidal characteristics related parameters used in the third experiment

\begin{tabular}{ll}
\hline \multicolumn{1}{c}{ Parameter } & Value \\
\hline Ratio of the data with Spring and workday characteristics $\eta_{s-\mathrm{w}}$ & 0.1 \\
\hline Ratio of the data with Spring and weekend characteristics $\eta_{s-\mathrm{h}}$ & 0.1 \\
\hline Ratio of the data with Spring but without tidal characteristics $\eta_{s-\mathrm{o}}$ & 0.8 \\
\hline Ratio of the data with workday but without seasonal characteristics $\eta_{o-\mathrm{w}}$ & 0.1 \\
\hline Ratio of the data with weekend but without seasonal characteristics $\eta_{o-\mathrm{h}}$ & 0.1 \\
\hline Ratio of the data with Spring and workday characteristics $\eta_{s-\mathrm{w}}$ & 0.8 \\
\hline
\end{tabular}

Table 26: Energy consumption of the three data placement strategies when the ratio of the data with tidal characteristics to the data without tidal characteristics is 2:8

\begin{tabular}{llll}
\hline $\begin{array}{l}\text { System } \\
\text { utilization }\end{array}$ & $\begin{array}{l}\text { Energy consumption of Kear } \\
(\mathrm{KJ})\end{array}$ & $\begin{array}{l}\text { Energy consumption of } \\
\text { Hadoop-default (KJ)[13] }\end{array}$ & $\begin{array}{l}\text { Energy consumption of } \\
\text { SEA(KJ) [12] }\end{array}$ \\
\hline 0.1 & 103759.5632 & 165958.2 & 107636.4326 \\
\hline 0.11 & 103784.1429 & 165966.084 & 107658.9143 \\
\hline 0.12 & 103808.7226 & 165973.968 & 107681.396 \\
\hline 0.13 & 103833.3023 & 165981.852 & 107703.8776 \\
\hline 0.14 & 103857.882 & 165989.736 & 107726.3593 \\
\hline 0.15 & 103882.4617 & 165997.62 & 107748.841 \\
\hline 0.16 & 103907.0413 & 166005.504 & 107771.3226 \\
\hline 0.17 & 103931.621 & 166013.388 & 107793.8043 \\
\hline 0.18 & 103956.2007 & 166021.272 & 107816.286 \\
\hline 0.19 & 103980.7804 & 166029.156 & 107838.7676 \\
\hline 0.2 & 104005.3601 & 166037.04 & 107861.2493 \\
\hline 0.21 & 104029.9398 & 166044.924 & 107883.7309 \\
\hline 0.22 & 104054.5194 & 166052.808 & 107906.2126 \\
\hline 0.23 & 104079.0991 & 166060.692 & 107928.6943 \\
\hline
\end{tabular}

This article is protected by copyright. All rights reserved. 


\begin{tabular}{llll}
\hline 0.24 & 104103.6788 & 166068.576 & 107951.1759 \\
\hline 0.25 & 104128.2585 & 166076.46 & 107973.6576 \\
\hline 0.26 & 104152.8382 & 166084.344 & 107996.1393 \\
\hline 0.27 & 104177.4179 & 166092.228 & 108018.6209 \\
\hline 0.28 & 104201.9976 & 166100.112 & 108041.1026 \\
\hline 0.29 & 104226.5772 & 166107.996 & 108063.5843 \\
\hline 0.3 & 104251.1569 & 166115.88 & 108086.0659 \\
\hline 0.31 & 104275.7366 & 166123.764 & 108108.5476 \\
\hline 0.32 & 104300.3163 & 166131.648 & 108131.0292 \\
\hline 0.33 & 104324.896 & 166139.532 & 108153.5109 \\
\hline 0.34 & 104349.4757 & 166147.416 & 108175.9926 \\
\hline 0.35 & 104374.0553 & 166155.3 & 108198.4742 \\
\hline 0.36 & 104398.635 & 166163.184 & 108220.9559 \\
\hline 0.37 & 104423.2147 & 166171.068 & 108243.4376 \\
\hline 0.38 & 104447.7944 & 166178.952 & 108265.9192 \\
\hline 0.39 & 104472.3741 & 166186.836 & 108288.4009 \\
\hline
\end{tabular}

Table 27 Common parameters used in the following three experiments

\begin{tabular}{|c|c|c|c|}
\hline Parameter & Value & Parameters & Value \\
\hline \multirow[t]{3}{*}{$\begin{array}{l}\text { Ratio of the data with Spring } \\
\text { characteristics } \eta_{s}\end{array}$} & \multirow[t]{3}{*}{0.2} & $\begin{array}{l}\text { Ratio of data with Spring and workday } \\
\text { characteristics } \eta_{s-\mathrm{w}}\end{array}$ & 0.3 \\
\hline & & $\begin{array}{l}\text { Ratio of data with Spring and weekend } \\
\text { characteristics } \eta_{s-\mathrm{h}}\end{array}$ & 0.3 \\
\hline & & $\begin{array}{l}\text { Ratio of data with Spring characteristics but } \\
\text { without tidal characteristics } \eta_{s-o}\end{array}$ & 0.4 \\
\hline \multirow[t]{3}{*}{$\begin{array}{l}\text { Ratio of the data without seasonal } \\
\text { characteristics } \eta_{o}\end{array}$} & \multirow[t]{3}{*}{0.2} & $\begin{array}{l}\text { Ratio of data without seasonal characteristics } \\
\text { but with workday characteristics } \eta_{o-w}\end{array}$ & 0.3 \\
\hline & & $\begin{array}{l}\text { Ratio of data without seasonal characteristics } \\
\text { but with weekend characteristics } \eta_{o-h}\end{array}$ & 0.3 \\
\hline & & $\begin{array}{l}\text { Ratio of data without seasonal characteristics } \\
\text { and tidal characteristics } \eta_{o-o}\end{array}$ & 0.4 \\
\hline Parameter & & Value & \\
\hline \multicolumn{2}{|c|}{$\begin{array}{l}\text { Ratio of the high speed disk utilization to the } \\
\text { system utilization }\end{array}$} & 2.0 & \\
\hline
\end{tabular}

Table 28 Energy consumption of the three data placement strategies when the ratio of the hot data to the cold data is 4:6

\begin{tabular}{llll}
\hline System & Energy consumption of Kear & Energy consumption of Hadoop-default & Energy consumption of SEA(KJ) \\
utilization & $(\mathrm{KJ})$ & $(\mathrm{KJ})[13]$ & {$[12]$} \\
\hline
\end{tabular}




\begin{tabular}{|c|c|c|c|}
\hline 0.1 & 95998.83104 & 165958.2 & 107636.4326 \\
\hline 0.11 & 96026.90742 & 165966.084 & 107658.9143 \\
\hline 0.12 & 96054.98381 & 165973.968 & 107681.396 \\
\hline 0.13 & 96083.06019 & 165981.852 & 107703.8776 \\
\hline 0.14 & 96111.13658 & 165989.736 & 107726.3593 \\
\hline 0.15 & 96139.21296 & 165997.62 & 107748.841 \\
\hline 0.16 & 96167.28934 & 166005.504 & 107771.3226 \\
\hline 0.17 & 96195.36573 & 166013.388 & 107793.8043 \\
\hline 0.18 & 96223.44211 & 166021.272 & 107816.286 \\
\hline 0.19 & 96251.5185 & 166029.156 & 107838.7676 \\
\hline 0.2 & 96279.59488 & 166037.04 & 107861.2493 \\
\hline 0.21 & 96307.67126 & 166044.924 & 107883.7309 \\
\hline 0.22 & 96335.74765 & 166052.808 & 107906.2126 \\
\hline 0.23 & 96363.82403 & 166060.692 & 107928.6943 \\
\hline 0.24 & 96391.90042 & 166068.576 & 107951.1759 \\
\hline 0.25 & 96419.9768 & 166076.46 & 107973.6576 \\
\hline 0.26 & 96448.05318 & 166084.344 & 107996.1393 \\
\hline 0.27 & 96476.12957 & 166092.228 & 108018.6209 \\
\hline 0.28 & 96504.20595 & 166100.112 & 108041.1026 \\
\hline 0.29 & 96532.28234 & 166107.996 & 108063.5843 \\
\hline 0.3 & 96560.35872 & 166115.88 & 108086.0659 \\
\hline 0.31 & 96588.4351 & 166123.764 & 108108.5476 \\
\hline 0.32 & 96616.51149 & 166131.648 & 108131.0292 \\
\hline 0.33 & 96644.58787 & 166139.532 & 108153.5109 \\
\hline 0.34 & 96672.66426 & 166147.416 & 108175.9926 \\
\hline 0.35 & 96700.74064 & 166155.3 & 108198.4742 \\
\hline 0.36 & 96728.81702 & 166163.184 & 108220.9559 \\
\hline 0.37 & 96756.89341 & 166171.068 & 108243.4376 \\
\hline 0.38 & 96784.96979 & 166178.952 & 108265.9192 \\
\hline 0.39 & 96813.04618 & 166186.836 & 108288.4009 \\
\hline
\end{tabular}

Table 29 Energy consumption of the three data placement strategies when the ratio of the hot data to the cold data is 3:7

\begin{tabular}{llll}
\hline $\begin{array}{l}\text { System } \\
\text { utilization }\end{array}$ & $\begin{array}{l}\text { Energy consumption of Kear } \\
(\mathrm{KJ})\end{array}$ & $\begin{array}{l}\text { Energy consumption of Hadoop-default } \\
(\mathrm{KJ})[13]\end{array}$ & $\begin{array}{l}\text { Energy consumption of SEA(KJ) } \\
{[12]}\end{array}$ \\
\hline 0.1 & 95998.83104 & 165958.2 & 97939.76338 \\
\hline 0.11 & 96026.90742 & 165966.084 & 97967.04052 \\
\hline 0.12 & 96054.98381 & 165973.968 & 97994.31766 \\
\hline 0.13 & 96083.06019 & 165981.852 & 98021.5948 \\
\hline
\end{tabular}

This article is protected by copyright. All rights reserved. 


\begin{tabular}{llll}
\hline 0.14 & 96111.13658 & 165989.736 & 98048.87194 \\
\hline 0.15 & 96139.21296 & 165997.62 & 98076.14907 \\
\hline 0.16 & 96167.28934 & 166005.504 & 98103.42621 \\
\hline 0.17 & 96195.36573 & 166013.388 & 98130.70335 \\
\hline 0.18 & 96223.44211 & 166021.272 & 98157.98049 \\
\hline 0.19 & 96251.5185 & 166029.156 & 98185.25763 \\
\hline 0.2 & 96279.59488 & 166037.04 & 98212.53477 \\
\hline 0.21 & 96307.67126 & 166044.924 & 98239.8119 \\
\hline 0.22 & 96335.74765 & 166052.808 & 98267.08904 \\
\hline 0.23 & 96363.82403 & 166060.692 & 98294.36618 \\
\hline 0.24 & 96391.90042 & 166068.576 & 98321.64332 \\
\hline 0.25 & 96419.9768 & 166076.46 & 98348.92046 \\
\hline 0.26 & 96448.05318 & 166084.344 & 98376.1976 \\
\hline 0.27 & 96476.12957 & 166092.228 & 98403.47473 \\
\hline 0.28 & 96504.20595 & 166100.112 & 98430.75187 \\
\hline 0.29 & 96532.28234 & 166107.996 & 98458.02901 \\
\hline 0.3 & 96560.35872 & 166115.88 & 98485.30615 \\
\hline 0.31 & 96588.4351 & 166123.764 & 98512.58329 \\
\hline 0.32 & 96616.51149 & 166131.648 & 98539.86043 \\
\hline 0.33 & 96644.58787 & 166139.532 & 98567.13756 \\
\hline 0.34 & 96672.66426 & 166147.416 & 98594.4147 \\
\hline 0.35 & 96700.74064 & 166155.3 & 98621.69184 \\
\hline 0.36 & 96728.81702 & 166163.184 & 98648.96898 \\
\hline 0.37 & 96756.89341 & 166171.068 & 98676.24612 \\
\hline 0.38 & 96784.96979 & 166178.952 & 98703.52326 \\
\hline 0.39 & 96813.04618 & 166186.836 & 98730.80039 \\
\hline & & & \\
\hline & & & \\
\hline & & & \\
\hline
\end{tabular}

Table 30 Energy consumption of the three data placement strategies when the ratio of the hot data to the cold data is 2:8

\begin{tabular}{llll}
\hline $\begin{array}{l}\text { System } \\
\text { utilization }\end{array}$ & $\begin{array}{l}\text { Energy consumption of Kear } \\
(\mathrm{KJ})\end{array}$ & $\begin{array}{l}\text { Energy consumption of Hadoop-default } \\
(\mathrm{KJ})[13]\end{array}$ & $\begin{array}{l}\text { Energy consumption of SEA(KJ) } \\
{[12]}\end{array}$ \\
\hline 0.1 & 95998.83104 & 165958.2 & 88231.10544 \\
\hline 0.11 & 96026.90742 & 165966.084 & 88261.97918 \\
\hline 0.12 & 96054.98381 & 165973.968 & 88292.85293 \\
\hline 0.13 & 96083.06019 & 165981.852 & 88323.72667 \\
\hline 0.14 & 96111.13658 & 165989.736 & 88354.60042 \\
\hline 0.15 & 96139.21296 & 165997.62 & 88385.47416 \\
\hline 0.16 & 96167.28934 & 166005.504 & 88416.3479 \\
\hline 0.17 & 96195.36573 & 166013.388 & 88447.22165 \\
\hline
\end{tabular}

This article is protected by copyright. All rights reserved. 


\begin{tabular}{llll}
\hline 0.18 & 96223.44211 & 166021.272 & 88478.09539 \\
\hline 0.19 & 96251.5185 & 166029.156 & 88508.96914 \\
\hline 0.2 & 96279.59488 & 166037.04 & 88539.84288 \\
\hline 0.21 & 96307.67126 & 166044.924 & 88570.71662 \\
\hline 0.22 & 96335.74765 & 166052.808 & 88601.59037 \\
\hline 0.23 & 96363.82403 & 166060.692 & 88632.46411 \\
\hline 0.24 & 96391.90042 & 166068.576 & 88663.33786 \\
\hline 0.25 & 96419.9768 & 166076.46 & 88694.2116 \\
\hline 0.26 & 96448.05318 & 166084.344 & 88725.08534 \\
\hline 0.27 & 96476.12957 & 166092.228 & 88755.95909 \\
\hline 0.28 & 96504.20595 & 166100.112 & 88786.83283 \\
\hline 0.29 & 96532.28234 & 166107.996 & 88817.70658 \\
\hline 0.3 & 96560.35872 & 166115.88 & 88848.58032 \\
\hline 0.31 & 96588.4351 & 166123.764 & 88879.45406 \\
\hline 0.32 & 96616.51149 & 166131.648 & 88910.32781 \\
\hline 0.33 & 96644.58787 & 166139.532 & 88941.20155 \\
\hline 0.34 & 96672.66426 & 166147.416 & 88972.0753 \\
\hline 0.35 & 96700.74064 & 166155.3 & 89002.94904 \\
\hline 0.36 & 96728.81702 & 166163.184 & 89033.82278 \\
\hline 0.37 & 96756.89341 & 166171.068 & 89064.69653 \\
\hline 0.38 & 96784.96979 & 166178.952 & 89095.57027 \\
\hline 0.39 & 96813.04618 & 166186.836 & 89126.44402 \\
\hline & & & \\
\hline
\end{tabular}

This article is protected by copyright. All rights reserved. 


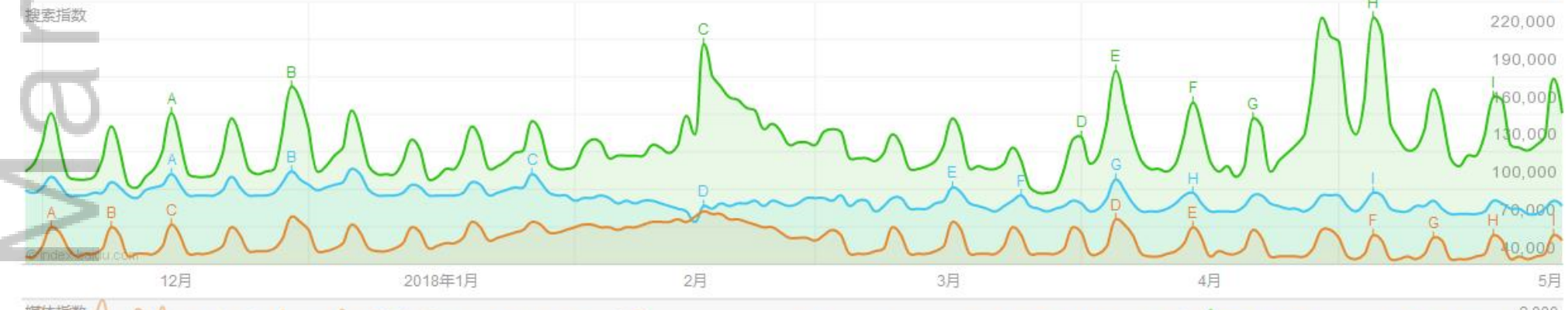

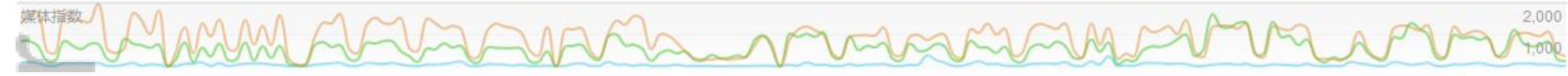
2011 CPE_6096_Fig.3 Varying curve of the search indexes of entertainment-related words in Baidu.tif 


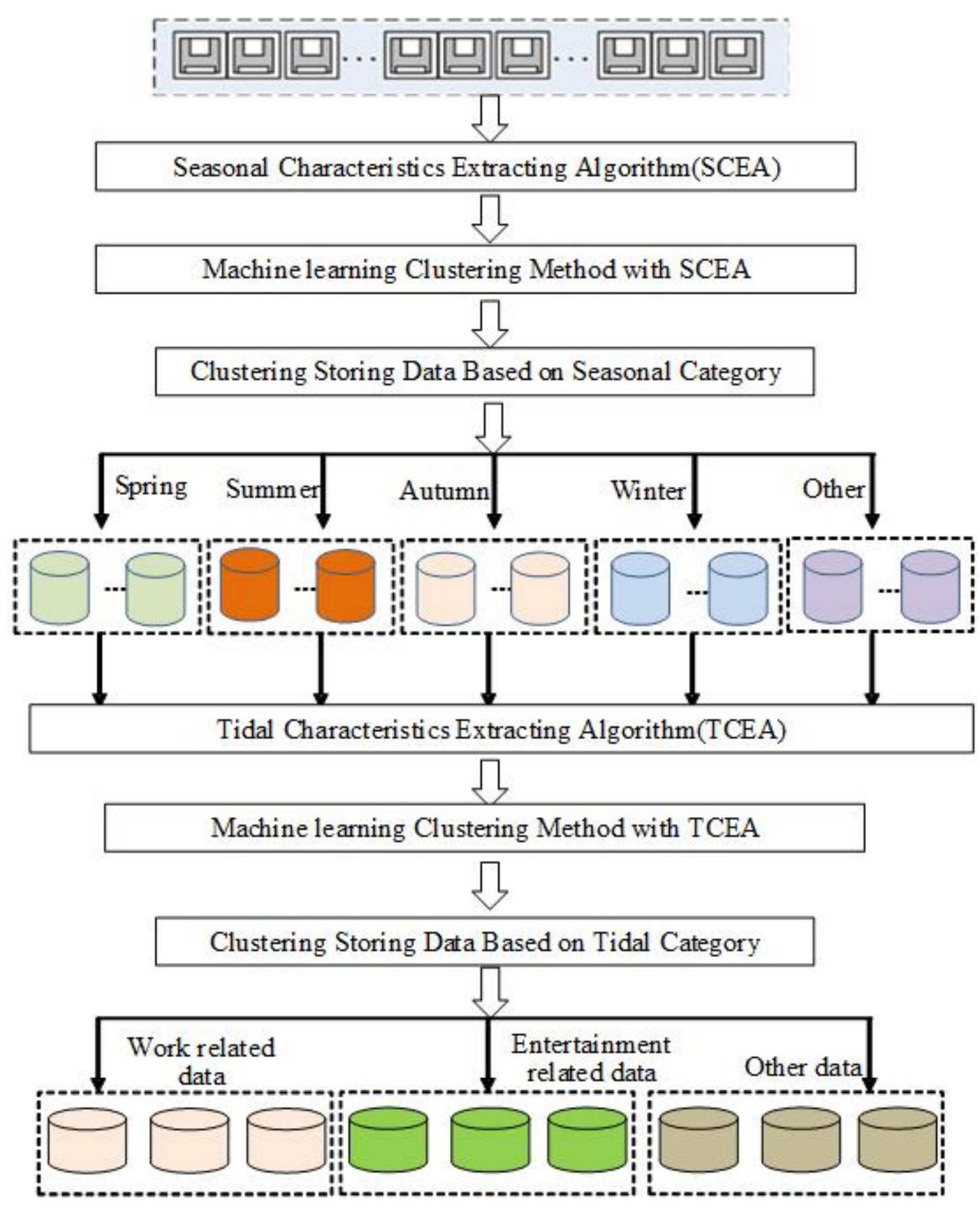

CPE_6096_Fig.4 Framework of K-ear.tif 

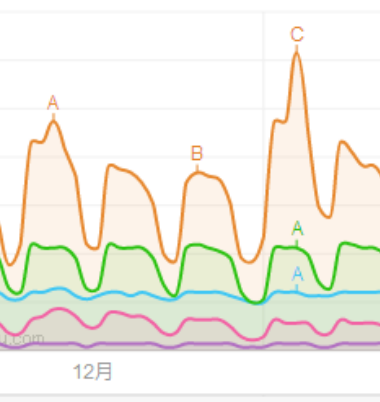

30,000

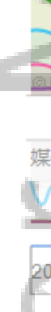

.

2018年1月

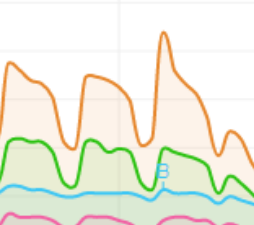

2月 3 月

3月

4月

体指数

2013

2015

$2 0 1 6 \longdiv { 1 6 } 2 0 1 7$

CPE_6096_Fig. 2 Varying curve of the search indexes of work-related words in Baidu.tif

This article is protected by copyright. All rights reserved. 


\section{University Library}

\section{- MINERVA \\ A gateway to Melbourne's research publications}

Minerva Access is the Institutional Repository of The University of Melbourne

Author/s:

You, X;Sun, T;Sun, D;Liu, X;Lv, X;Buyya, R

Title:

K-ear: Extracting data access periodic characteristics for energy-aware data clustering and storing in cloud storage systems

Date:

2020-11-30

Citation:

You, X., Sun, T., Sun, D., Liu, X., Lv, X. \& Buyya, R. (2020). K-ear: Extracting data access periodic characteristics for energy-aware data clustering and storing in cloud storage systems. CONCURRENCY AND COMPUTATION-PRACTICE \& EXPERIENCE, 33 (9), https:// doi.org/10.1002/cpe.6096.

Persistent Link:

http://hdl.handle.net/11343/276664 